\title{
Staying well connected - Lithistid sponges on seamounts
}

\author{
MERRICK EKINS ${ }^{1}$, DIRK ERPENBECK ${ }^{2}$, GERT WÖRHEIDE ${ }^{3}$ AND JOHN N. A. HOOPER ${ }^{1,4}$ \\ ${ }^{1}$ Queensland Museum, PO Box 3300, South Brisbane 4101, Brisbane, Queensland, Australia, ${ }^{2}$ Department of Earth and \\ Environmental Sciences and GeoBio-Center, Ludwig-Maximilians-Universität München, Richard-Wagner-Straße 10, 80333 \\ München, Germany, ${ }^{3}$ SNSB - Bavarian State Collections for Palaeontology and Geology, Richard-Wagner-Straße 10, 80333 \\ München, Germany, ${ }^{4}$ Eskitis Institute for Drug Discovery, Griffith University, Brisbane 4111, Queensland, Australia
}

\begin{abstract}
Three species of lithistid sponges, Neoaulaxinia zingiberadix, Isabella mirabilis and Neoschrammeniella fulvodesmus were collected from deep seamounts off New Caledonia to address questions about their population structure, gene flow and the relative contribution of sexual and asexual reproductive strategies to their populations. The sponges were tested by sequencing the ITS (internal transcribed spacer) and CO1 regions of their genomes. These rare and presumably ancient sponges have a distribution restricted to seamounts in the south-western Pacific. Deep seamounts represent geographically separated islands. Although the sponges could be expected to have sexual reproduction restricted to near neighbours due to low sexual dispersal opportunities via larvae, this study found surprisingly high levels of gene flow between the seamounts. Amongst the specimens of N. zingiberadix taken from two seamounts there was no population structure; CO1 resulted in identical genotypes. For the population structure within $\mathrm{N}$. fulvodesmus, as revealed by ITS, most of the variation was within each individual from the six seamounts on which it occurred and $\mathrm{CO} 1$ revealed no difference between individuals or seamounts. The third species I. mirabilis showed four genotypes based on $\mathrm{CO}_{1}$, which were distributed across all the seamounts. Indirect measures of different species showed a range of reproductive strategies from asexual to sexual, but with much higher connection between seamounts than previously thought. Individual seamounts did not show a separate population structure as one might expect from 'islands'. The conclusion must be that these sponges have mechanisms to attain greater dispersal than previously thought.
\end{abstract}

Keywords: Lithistid, population genetics, seamounts, gene flow, dispersal, reproduction, Neoaulaxinia zingiberadix, Isabella mirabilis, Neoschrammeniella fulvodesmus

Submitted 23 January 2015; accepted 19 May 2015; first published online 22 June 2015

\section{INTRDDUCTION}

Sponges occupy widely divergent biogeographic regions. This may be a testament to their ability to colonize over long distances, or their ability to adapt and evolve to suit changing environments. Yet the mechanisms whereby they do this, in particular the relative importance of both sexual and asexual reproduction strategies, is largely unknown for many sponges. Sponges found on seamounts are isolated, submerged and independent offering an opportunity to test various possibilities.

Sponges are known to use both sexual and asexual reproductive strategies to survive and prosper (Kaye, 1990) and both sexual and asexual components to enable long-range dispersal and colonization of new habitats (see overview in Maldonado \& Riesgo, 2008). Active locomotion of entire individual sponges has been observed in response to environmental factors (Bond \& Harris, 1988; Maldonado \& Uriz, 1999b), but such an ability, used to readjust the sponge position at the microhabitat scale (displacements from millimetres to centimetres) cannot account for population dispersal. Dispersal of sexual stages is generally assumed to involve the

Corresponding author:

M. Ekins

Email: m.ekins@qm.qld.gov.au transmission of a sexual form, such as detached tissue still undergoing post fertilization development (Maldonado \& Uriz, 1999b), sperm and eggs, or a swimming or crawling larval stage that lasts for only a few days (Maldonado \& Riesgo, 2008). These are potentially transmitted via currents, waves, storms or using vectors such as fish or echinoderms. Asexual reproduction has also been observed and includes abiotic factors such as physical fragmentation (Wulff, 1991) and biotic factors such as fission, budding (e.g. Teixido et al., 2006; Ereskovsky \& Tokina, 2007) and gemmule formation (Sarà et al., 2002). Both strategies have been shown to occur in individual sponges concurrently (Leong \& Pawlik, 2010) or successionally (Ereskovsky \& Tokina, 2007). It is the sponges' ability to use both of these strategies synergistically, that improves their dispersal ability (Maldonado \& Uriz, 1999a). The formation of external buds of hexactinellids in the stable environment of Antarctic waters (Teixido et al., 2006) may enable the formation of a stable clonal population in the immediate vicinity. The formation of gemmules generally associated with freshwater sponges also occur in some marine sponges (Sarà et al., 2002). However, these gemmules are expected to have dispersal abilities on par with sexual propagules. The formation of bubble-like buds in Oscarella spp. (Ereskovsky \& Tokina, 2007) and Leucetta changosensis Dendy, 1913 (Wörheide et al., 2008) shows a previously unnoticed far-reaching asexual dispersal mechanism. Merging of conspecific sponges (Wulff, 1990) also provides 
another possible asexual reproduction mechanism which is not usually accompanied by meiotic recombination but carried out by totipotent somatic cells in sponges.

Colonization of new locations by asexual stages may, alternatively, be more commonly due to the dispersal of fragments or entire sponges, via waves, currents, storms and cyclones, or as a passenger on substrates such as debris. There are several characteristics that may indicate an asexual or clonal population including frequent recovery of genotypes, correlation between different independent markers, linkage disequilibrium and the absence of sexual reproductive structures. Direct observation of deep sea sponge dispersal is not currently feasible, although observations indicate it can be done at great expense (Teixido et al., 2006). In other sessile invertebrates such as corals, asexual reproduction is common and is an adaptation that succeeds both in moderate to high levels of disturbance (Le GoffVitry et al., 2004; Miller \& Ayre, 2004) and stable environments (Foster et al., 2007) as it enables well-adapted individuals (Ayre \& Willis, 1988) to rapidly colonize environments or maintain dominance in environments unfavourable for sexual reproduction (Wulff, 1991; Hughes et al., 1992). Branching corals, which have relatively fragile morphology, are more likely to have high asexual components (Le Goff-Vitry et al., 2004) than more stable massive corals (Foster et al., 2007). By contrast, the coral excavating sponge Cliona delitrix Pang, 1973 was shown to not have clonal propagation, as was expected, resulting from coral breakage (Zilberberg et al., 2006b).

Research has been carried out exploring the contributions of sexual and asexual recruitment on sponges from physical observations (Battershill \& Bergquist, 1990; Wulff, 1991) However, other indirect methods to assess the relative input of sexual and asexual reproductive forms in the dispersal process have been used including histocompatibility (Neigel \& Avise, 1983), allozymes (Zilberberg et al., 2006a; Whalan et al., 2008), mitochondrial DNA such as CO1 sequence data (Duran et al., 2004a; Whalan et al., 2008; Dailianis et al., 2011), microsatellites (Duran et al., 2002, 2004C; Blanquer et al., 2005), introns (Bentlage \& Worheide, 2007; Wörheide et al., 2008) and nuclear ribosomal internal transcribed spacer (ITS) sequence data (Lopez et al., 2002; Wörheide et al., 2002a, b; Duran et al., 2004a; Nichols \& Barnes, 2005; Duran \& Rützler, 2006; Hoshino et al., 2008). Within an individual sponge, the multiple copies of the ITS have been suggested to be not yet homogenized by concerted evolution (Duran et al., 2004a). However, intra-genomic polymorphisms (IGPs) within individual sponges have been detected in about half of sponge species tested (Duran et al., 2004a; Wörheide et al., 2004). This indicates that the variation occurring within individuals may be responsible for much of the variation attributed to different species, genera and even families. Indeed in phylogenetic studies the populations should always be screened before phylogenetic comparisons are carried out between different taxa. Other researchers have also detected ITS polymorphisms ranging from single base changes (Lopez et al., 2002), several nucleotides (Duran et al., 2004a) and variable sites (Schmitt et al., 2005) through to examples of up to 13 sequence types per individual (Wörheide et al., 2004). Intra-genomic variation is the presence of multiple genotypes within an individual sponge. However, it may also be an example of multiple sponges forming of chimeras (Blanquer \& Uriz, 2011).
Population research in sponges has largely concentrated on biogeography (Nichols \& Barnes, 2005), the movement of species into or out of the Mediterranean Sea (Duran et al., 2004a), survival in the South Pacific (Wörheide et al., 2002a) and ecological specialization (Duran \& Rützler, 2006). Much of the work on sponge population genetics has shown genetic differentiation at geographic scales (Klautau et al., 1999; Duran et al., 2004a, b; Bentlage \& Worheide, 2007; Hoshino et al., 2008; Wörheide et al., 2008; Xavier et al., 2010); for a review see Uriz \& Turon (2012). By comparison, only a small amount of research has been carried out on small scales as would occur on a seamount. Two Mediterranean studies on Crambe crambe (Schmidt, 1862) (Calderon et al., 2007) and Scopalina lophyropoda Schmidt, 1862 (Blanquer et al., 2009) showed genetic structure at this small scale consistent with what one would expect from a poorly dispersing sexual stage, although this result could be confused by the presence of chimeric individuals (Blanquer \& Uriz, 2011). Seamounts have been predicted to have large amounts of population structure because of spatial separation and limited dispersal mechanisms coupled with high levels of asexual reproduction (Richer de Forges et al., 2000; Samadi et al., 2006). Seamounts are believed to be particularly susceptible to genetic drift due to their small size (Le Goff-Vitry et al., 2004). Seamounts are not ephemeral and, coupled with low gene flow due to distance and hydraulics, structure within populations would be expected (Samadi et al., 2006). However, research on crustaceans collected from New Caledonian seamounts showed that previous perceived high endemism (Richer de Forges et al., 2000) was not the case for seamounts. Instead the crustaceans had high levels of gene flow between the seamounts. The only exception in fact was a gastropod that had limited larval dispersal (Samadi et al., 2006).

This study focuses on three rare, deep and isolated species of lithisitid ('rock') sponges from deep seamounts in New Caledonia that are several million years old (Richer de Forges et al., 2000), Neoaulaxinia zingiberadix (Kelly, 2007), Isabella mirabilis (Schlacher-Hoenlinger et al., 2005) and Neoschrammeniella fulvodesmus (Lévi \& Lévi, 1983). Lithistids on these seamounts may be relict fauna from the Mesozoic Era (Lévi, 1991), surviving on refuge habitat (Samadi et al., 2007). Lithistids are found in most parts of the world at great depths. They are believed to be long lived and slow growing and, as such, display $\mathrm{K}$ reproductive strategies such as ovipary (Reiswig, 1973; Fromont \& Bergquist, 1994). Lithistid sponges constitute a polyphyletic collection of disparate families and genera grouped together by the common presence of desmas. However, the latter remains significantly unresolved based on morphology, and still awaits independent datasets to support or refute morphometric hypotheses (e.g. Pisera \& Lévi, 2002). Indeed because lithistids represent an artificial group split into several unrelated orders (Schuster et al., 2015), it is likely there will be varying reproductive strategies within the lithistids. Only one study on reproduction in lithistids has been reported (Maldonado \& Bergquist, 2002; Maldonado \& Riesgo, 2008). and this is based purely on studies from Theonella, which has been recently revised to Astrophorida (Hall et al., 2014). The one study indicated the Theonella is gonochoric and oviparous which means they release zygotes or early embryos that develop externally (Maldonado \& Bergquist, 2002; Maldonado \& Riesgo, 2008). However, these reproductive 
modes are more likely to be strategies to suit the current environmental conditions (Ereskovsky, 2010). Lithistids have been reported to have either parenchymella or clavablastula larval types, with free swimming planktonic or crawling demersal larval stages (Hooper \& Van Soest, 2002). Some of the larval stages are thought to survive for only a few hours and up to 3 weeks (Maldonado \& Bergquist, 2002). However, other astrophorid sponge genera Thoosa and Alectona produce an uncilliated hoplitomella larvae, which shows great dispersal characteristics, using its protruding spicules to float for long periods of time (Maldonado \& Bergquist, 2002; Borchiellini et al., 2004; Maldonado, 2004; Bautista-Guerrero et al., 2010). The sexual structures and larval stages have not been observed for the three species used in this research. However, because of the limited dispersal characteristics of sponge larvae in general, sponge populations have been thought to have local low genetic variability, high genetic structure and incipient species (Jablonski, 1986; Jackson, 1986; Wörheide et al., 2005). Nonetheless, due to the large dispersal characteristics of some deep sea astrophorids, these lithistids may indeed have greater dispersal characteristics but require specific environmental conditions to survive that may occur only on deep seamounts.

The depths from which the lithistid sponges used in this study are recovered (270-1032 $\mathrm{m}$ depth range) would have enabled survival during the glacial ice age without having to move down the slope to colder deeper water. In addition, these populations are unlikely to have suffered disturbance from human activities, few surface climatic effects, little predation, and since they are on seamounts they are unlikely to have been affected by continental erosion. The main aim of this research is to evaluate if there are discrete populations of these three lithistid species on different seamount 'islands' up to almost $200 \mathrm{~km}$ apart, with the abyssal floor between these seamounts at $4000 \mathrm{~m}$ depth. Previous research on fauna from seamounts has found highly localized species distributions and apparent speciation between island groups or ridge systems in the south-western Pacific (Richer de Forges et al. 2000). This research is the first attempt to investigate if specimens of an individual sponge species from different seamounts belong to genetically distinct populations (genotypes), or are truly widespread as their present taxonomy suggests. Further we will use direct (i.e. genotypes) and indirect (i.e. linkage disequilibrium) tests to measure the relative components of sexual and asexual reproduction within this species of sponge.

\section{MATERIALS AND METHODS}

\section{Sampling, DNA extraction and sequencing}

The lithistid specimens sponges were collected by dredge from deep seamounts off New Caledonia across 10 sites at depths ranging from 270 to $1032 \mathrm{~m}$. The only specimens collected by trawling of up to $14 \mathrm{~km}$ transect length were the specimens from Eponge North. The sponges were frozen immediately following collection. All specimens were registered at the Queensland Museum, registration numbers between QM G329756 to QM G331837 (Table 1). The species were identified morphologically by soaking thin tissue section in Histo-Clear II (National Diagnostics, USA) for $24 \mathrm{~h}$ before mounting in Histomount (National Diagnostics, USA), and examined under an Olympus $\mathrm{BH}-1$ light microscope.
Spicules were dissociated by dissolving sponge tissue in boiling nitric acid, rinsed in water and resuspended in absolute ethanol. They were then either mounted in Durcupan ACM (Sigma-Aldrich, Australia) and examined under an Olympus BH-1 light microscope, or ignited on an SEM stub and examined using a Hitachi H1ooo SEM.

For molecular work, samples from 131 specimens were taken by cutting a $5 \mathrm{~mm}^{3}$ section of sponge tissue from the middle of the specimen. DNA was extracted using a DNAeasy ${ }^{\circledR}$ Tissue Kit (Qiagen, Australia) according to the manufacturer's instructions. The ribosomal internal transcribed spacer (ITS) region, including the entire ITS $_{1}, 5.8 \mathrm{~S}$ rRNA and ITS2 regions, was amplified using primers RA2: GTCCCTGCCCTTTGTACACA and ITS2.2: CCTGGTT AGTTTCTTTTCCTCCGC. PCR amplifications were carried out in a $25 \mu \mathrm{L}$ volume reaction, with 1 unit of HotmasterTaq $^{\circledR}$ (Eppendorf), $200 \mu \mathrm{M}$ of mixed dNTPs and $10 \mu \mathrm{M}$ of each primer. Reaction conditions consisted of a denaturing step of $2 \mathrm{~min}$ at $95^{\circ} \mathrm{C}, 35$ cycles of $20 \mathrm{~s}$ at $95^{\circ} \mathrm{C}$, $10 \mathrm{~s}$ at $58^{\circ} \mathrm{C}$, and $1 \mathrm{~min}$ at $65^{\circ} \mathrm{C}$, followed by a final extension of $10 \mathrm{~min}$ at $65^{\circ} \mathrm{C}$. The PCR product was electrophoresed on a $0.5 \%$ agarose gel for $1 \mathrm{~h}$ at 90 volts, the single band excised, and the $1 \mathrm{~kb}$ product purified using Perfectprep Gel Cleanup kit (Eppendorf, Germany). The PCR product was then cloned using pDrive Vector (Qiagen) following the manufacturer's instructions, modified to half concentration, i.e. $\frac{1}{2} \mathrm{X}$ reaction and the addition of $800 \mu \mathrm{L}$ of $37^{\circ} \mathrm{C}$ SOC during transformations. Successful recombinants were selected and grown in liquid culture and plasmid DNA isolated via alkaline lysis minipreps (Sambrook et al., 1989). Between two and seven plasmids per specimen were sequenced directly in $12 \mu \mathrm{L}$ reactions containing $3 \mu \mathrm{L}$ of $5 \times$ BigDye Terminator Buffer (Applied Biosystems, Australia), o.8 $\mu \mathrm{M}$ of either $\mathrm{M}_{13} \mathrm{~F}$ or $\mathrm{T} 7$ universal primer, $200 \mathrm{ng}$ of plasmid DNA, and $1 \mu \mathrm{L}$ Big Dye Terminator Mix v3.1 (Applied Biosystems, Australia). Reactions were denatured for $5 \mathrm{~min}$ at $94^{\circ} \mathrm{C}$, followed by 30 cycles of $10 \mathrm{~s}$ at $96^{\circ} \mathrm{C}, 5 \mathrm{~s}$ at $50^{\circ} \mathrm{C}$, and $4 \mathrm{~min}$ at $60^{\circ} \mathrm{C}$. The sequencing reactions were precipitated using $75 \mu \mathrm{L}$ of a $70 \%$ ethanol solution with $0.0002 \mathrm{M} \mathrm{MgSO}_{4}$ and centrifuged at $3600 \mathrm{rpm}$ for $15 \mathrm{~min}$ before the supernatant was discarded. Sequencing was carried out on an ABI $3730 \times 196$ capillary automated DNA sequencer. Sequences were aligned using Sequencher ${ }^{\mathrm{TM}} 4.5$ (Genecodes) and BIOEDIT 7.0.4.1 (Hall, 1999).

$\mathrm{CO}_{1}$ data has been obtained during the barcoding of the Queensland Museum sponge collection in the course of the Sponge Barcoding Project (www.spongebarcoding.org) (Wörheide \& Erpenbeck, 2007). The DNA extraction followed a plate-based extraction method (Vargas et al., 2010). Fragments of $\mathrm{CO}_{1}$ standard barcoding fragment were amplified using the degenerated barcoding primers dgLCO1490 and dgHCO2198 (Meyer et al., 2005) with annealing temperature of $43^{\circ} \mathrm{C}$. The PCR products were purified with ExoSAP-IT (Affymetrix) or standard Ammonium Acetate-Ethanol precipitation before cycle sequencing both strands with the Big Dye Terminator Mix v3.1 (Applied Biosystems, Australia) following the manufacturer's protocol and sequencing on an $\mathrm{ABI}$ 3730 automated sequencer. The poriferan origin of the sequences was checked by a BLAST search against the NCBI GenBank nr database (http://www.ncbi.nlm.nih.gov/). Sequences were base called and assembled to a 563 bp alignment in CodonCode Aligner v3.7.1.1 and subsequently aligned in Sea-View 4 (Galtier et al., 1996) using Muscle 3.6 
Table 1. List of the species and the collection data of all of the specimens used in this study.

\begin{tabular}{|c|c|c|c|c|c|c|c|c|}
\hline $\begin{array}{l}\text { Queensland Museum } \\
\text { Registration }\end{array}$ & Species & Location & $\begin{array}{l}\text { Depth } \\
\text { Deploy (m) }\end{array}$ & $\begin{array}{l}\text { Depth } \\
\text { Retrieve (m) }\end{array}$ & $\begin{array}{l}\text { Latitude } \\
\text { (Degrees) }\end{array}$ & $\begin{array}{l}\text { Longitude } \\
\text { (Degrees) }\end{array}$ & ITS & $\mathrm{CO}_{1}$ \\
\hline $\mathrm{G}_{329825}$ & N. clavata & Jumeau East & 470 & 621 & $23.715 \mathrm{~S}$ & $168.257 \mathrm{E}$ & ITS & $\mathrm{CO}_{1}$ \\
\hline $\mathrm{G}_{329756}$ & N. fulvodesmus & Jumeau East & 470 & 621 & $23.715 \mathrm{~S}$ & $168.257 \mathrm{E}$ & ITS & $\mathrm{CO}_{1}$ \\
\hline $\mathrm{G}_{329757}$ & N. fulvodesmus & Jumeau East & 470 & 621 & $23.715 \mathrm{~S}$ & $168.257 \mathrm{E}$ & ITS & $\mathrm{CO}_{1}$ \\
\hline $\mathrm{G}_{329758}$ & N. fulvodesmus & Jumeau East & 470 & 621 & $23.715 \mathrm{~S}$ & $168.257 \mathrm{E}$ & ITS & \\
\hline $\mathrm{G}_{329759}$ & N. fulvodesmus & Jumeau East & 470 & 621 & $23.715 \mathrm{~S}$ & $168.257 \mathrm{E}$ & ITS & \\
\hline $\mathrm{G}_{329760}$ & N. fulvodesmus & Jumeau East & 470 & 621 & $23.715 \mathrm{~S}$ & $168.257 \mathrm{E}$ & ITS & \\
\hline $\mathrm{G}_{329761}$ & N. fulvodesmus & Jumeau East & 470 & 621 & $23.715 \mathrm{~S}$ & $168.257 \mathrm{E}$ & ITS & $\mathrm{CO}_{1}$ \\
\hline $\mathrm{G}_{329762}$ & N. fulvodesmus & Jumeau East & 470 & 621 & $23.715 \mathrm{~S}$ & $168.257 \mathrm{E}$ & ITS & $\mathrm{CO}_{1}$ \\
\hline$G_{329763}$ & N. fulvodesmus & Jumeau East & 470 & 621 & $23.715 \mathrm{~S}$ & $168.257 \mathrm{E}$ & ITS & \\
\hline $\mathrm{G}_{329764}$ & N. fulvodesmus & Jumeau East & 470 & 621 & $23.715 \mathrm{~S}$ & $168.257 \mathrm{E}$ & ITS & \\
\hline$G_{329765}$ & N. fulvodesmus & Jumeau East & 470 & 621 & $23.715 \mathrm{~S}$ & $168.257 \mathrm{E}$ & ITS & \\
\hline$G_{329766}$ & N. fulvodesmus & Zorro South & 623 & 691 & $25.400 \mathrm{~S}$ & $168.333 \mathrm{E}$ & ITS & \\
\hline $\mathrm{G}_{329767}$ & N. fulvodesmus & Zorro South & 623 & 691 & $25.400 \mathrm{~S}$ & $168.333 \mathrm{E}$ & ITS & \\
\hline $\mathrm{G}_{329768}$ & N. fulvodesmus & Zorro South & 623 & 691 & $25.400 \mathrm{~S}$ & $168.333 \mathrm{E}$ & ITS & \\
\hline$G_{329769}$ & N. fulvodesmus & Zorro South & 623 & 691 & $25.400 \mathrm{~S}$ & $168.333 \mathrm{E}$ & ITS & $\mathrm{CO}_{1}$ \\
\hline $\mathrm{G}_{329770}$ & N. fulvodesmus & Zorro South & 623 & 691 & $25.400 \mathrm{~S}$ & $168.333 \mathrm{E}$ & ITS & \\
\hline $\mathrm{G}_{329771}$ & N. fulvodesmus & Zorro South & 623 & 691 & $25.400 \mathrm{~S}$ & $168.333 \mathrm{E}$ & ITS & \\
\hline $\mathrm{G}_{329772}$ & N. fulvodesmus & Zorro South & 623 & 691 & $25.400 \mathrm{~S}$ & $168.333 \mathrm{E}$ & ITS & \\
\hline $\mathrm{G}_{329773}$ & N. fulvodesmus & Zorro South & 623 & 691 & $25.400 \mathrm{~S}$ & $168.333 \mathrm{E}$ & ITS & \\
\hline $\mathrm{G}_{329774}$ & N. fulvodesmus & Zorro South & 623 & 691 & $25.400 \mathrm{~S}$ & $168.333 \mathrm{E}$ & ITS & \\
\hline$G_{329775}$ & N. fulvodesmus & Zorro South & 623 & 691 & $25.400 \mathrm{~S}$ & $168.333 \mathrm{E}$ & ITS & \\
\hline $\mathrm{G}_{329776}$ & N. fulvodesmus & Zorro North & 666 & 1000 & $25.344 \mathrm{~S}$ & $168.309 \mathrm{E}$ & ITS & \\
\hline $\mathrm{G}_{329777}$ & N. fulvodesmus & Zorro North & 666 & 1000 & $25.344 \mathrm{~S}$ & $168.309 \mathrm{E}$ & ITS & \\
\hline $\mathrm{G}_{329778}$ & N. fulvodesmus & Zorro North & 666 & 1000 & $25.344 \mathrm{~S}$ & $168.309 \mathrm{E}$ & ITS & \\
\hline $\mathrm{G}_{329779}$ & N. fulvodesmus & Zorro North & 666 & 1000 & $25.344 \mathrm{~S}$ & $168.309 \mathrm{E}$ & ITS & \\
\hline $\mathrm{G}_{329780}$ & N. fulvodesmus & Zorro North & 666 & 1000 & $25.344 \mathrm{~S}$ & $168.309 \mathrm{E}$ & ITS & \\
\hline $\mathrm{G}_{329781}$ & N. fulvodesmus & Zorro North & 666 & 1000 & $25.344 \mathrm{~S}$ & $168.309 \mathrm{E}$ & ITS & \\
\hline $\mathrm{G}_{329782}$ & N. fulvodesmus & Zorro North & 666 & 1000 & $25.344 \mathrm{~S}$ & $168.309 \mathrm{E}$ & ITS & \\
\hline $\mathrm{G}_{329783}$ & N. fulvodesmus & Zorro North & 666 & 1000 & $25.344 \mathrm{~S}$ & $168.309 \mathrm{E}$ & ITS & \\
\hline $\mathrm{G}_{329784}$ & N. fulvodesmus & Zorro North & 666 & 1000 & $25.344 \mathrm{~S}$ & $168.309 \mathrm{E}$ & ITS & \\
\hline $\mathrm{G}_{329785}$ & N. fulvodesmus & Zorro North & 666 & 1000 & $25.344 \mathrm{~S}$ & $168.309 \mathrm{E}$ & ITS & \\
\hline $\mathrm{G}_{329786}$ & N. fulvodesmus & Eponge North & 530 & 540 & $24.887 \mathrm{~S}$ & $168.364 \mathrm{E}$ & ITS & \\
\hline $\mathrm{G}_{329787}$ & N. fulvodesmus & Eponge North & 530 & 540 & $24.887 \mathrm{~S}$ & $168.364 \mathrm{E}$ & ITS & \\
\hline $\mathrm{G}_{329788}$ & N. fulvodesmus & Eponge North & 530 & 540 & $24.887 \mathrm{~S}$ & $168.364 \mathrm{E}$ & ITS & \\
\hline $\mathrm{G}_{329789}$ & N. fulvodesmus & Eponge North & 530 & 540 & $24.887 \mathrm{~S}$ & $168.364 \mathrm{E}$ & ITS & \\
\hline$G_{329790}$ & N. fulvodesmus & Eponge North & 530 & 540 & $24.887 \mathrm{~S}$ & $168.364 \mathrm{E}$ & ITS & \\
\hline $\mathrm{G}_{329791}$ & N. fulvodesmus & Eponge North & 530 & 540 & $24.887 \mathrm{~S}$ & $168.364 \mathrm{E}$ & ITS & \\
\hline $\mathrm{G}_{329792}$ & N. fulvodesmus & Eponge North & 530 & 540 & $24.887 \mathrm{~S}$ & $168.364 \mathrm{E}$ & ITS & \\
\hline G329793 & N. fulvodesmus & Eponge North & 530 & 540 & $24.887 \mathrm{~S}$ & $168.364 \mathrm{E}$ & ITS & \\
\hline $\mathrm{G}_{329794}$ & N. fulvodesmus & Eponge North & 530 & 540 & $24.887 \mathrm{~S}$ & $168.364 \mathrm{E}$ & ITS & \\
\hline$G_{329795}$ & N. fulvodesmus & Eponge North & 530 & 540 & $24.887 \mathrm{~S}$ & $168.364 \mathrm{E}$ & ITS & \\
\hline $\mathrm{G}_{329796}$ & N. fulvodesmus & Eponge South & 518 & 586 & $24.937 \mathrm{~S}$ & $168.361 \mathrm{E}$ & ITS & \\
\hline $\mathrm{G}_{329797}$ & N. fulvodesmus & Eponge South & 518 & 586 & $24.937 \mathrm{~S}$ & $168.361 \mathrm{E}$ & ITS & \\
\hline $\mathrm{G}_{329798}$ & N. fulvodesmus & Eponge South & 518 & 586 & $24.937 \mathrm{~S}$ & $168.361 \mathrm{E}$ & ITS & \\
\hline $\mathrm{G}_{329799}$ & N. fulvodesmus & Eponge South & 518 & 586 & $24.937 \mathrm{~S}$ & $168.361 \mathrm{E}$ & ITS & \\
\hline $\mathrm{G}_{329800}$ & N. fulvodesmus & Eponge South & 518 & 586 & $24.937 \mathrm{~S}$ & $168.361 \mathrm{E}$ & ITS & \\
\hline$G_{329801}$ & N. fulvodesmus & Eponge South & 518 & 586 & $24.937 \mathrm{~S}$ & $168.361 \mathrm{E}$ & ITS & \\
\hline $\mathrm{G}_{329802}$ & N. fulvodesmus & Eponge South & 518 & 586 & $24.937 \mathrm{~S}$ & $168.361 \mathrm{E}$ & ITS & \\
\hline $\mathrm{G}_{329803}$ & N. fulvodesmus & Eponge South & 518 & 586 & $24.937 \mathrm{~S}$ & $168.361 \mathrm{E}$ & ITS & \\
\hline $\mathrm{G}_{329804}$ & N. fulvodesmus & Eponge South & 518 & 586 & $24.937 \mathrm{~S}$ & $168.361 \mathrm{E}$ & ITS & \\
\hline $\mathrm{G}_{329805}$ & N. fulvodesmus & Eponge South & 518 & 586 & $24.937 \mathrm{~S}$ & $168.361 \mathrm{E}$ & ITS & \\
\hline $\mathrm{G}_{329806}$ & N. fulvodesmus & Kaimon Maru & 600 & 896 & $24.756 \mathrm{~S}$ & $168.104 \mathrm{E}$ & ITS & $\mathrm{CO}_{1}$ \\
\hline $\mathrm{G}_{329807}$ & N. fulvodesmus & Kaimon Maru & 600 & 896 & $24.756 \mathrm{~S}$ & $168.104 \mathrm{E}$ & ITS & \\
\hline $\mathrm{G}_{329808}$ & N. fulvodesmus & Kaimon Maru & 600 & 896 & $24.756 \mathrm{~S}$ & $168.104 \mathrm{E}$ & ITS & \\
\hline $\mathrm{G}_{329809}$ & N. fulvodesmus & Kaimon Maru & 600 & 896 & $24.756 \mathrm{~S}$ & $168.104 \mathrm{E}$ & ITS & \\
\hline $\mathrm{G}_{329810}$ & N. fulvodesmus & Kaimon Maru & 600 & 896 & $24.756 \mathrm{~S}$ & $168.104 \mathrm{E}$ & ITS & \\
\hline $\mathrm{G}_{329811}$ & N. fulvodesmus & Kaimon Maru & 600 & 896 & $24.756 \mathrm{~S}$ & $168.104 \mathrm{E}$ & ITS & \\
\hline $\mathrm{G}_{329812}$ & N. fulvodesmus & Kaimon Maru & 600 & 896 & $24.756 \mathrm{~S}$ & $168.104 \mathrm{E}$ & ITS & \\
\hline $\mathrm{G}_{329813}$ & N. fulvodesmus & Kaimon Maru & 600 & 896 & $24.756 \mathrm{~S}$ & $168.104 \mathrm{E}$ & ITS & $\mathrm{CO}_{1}$ \\
\hline $\mathrm{G}_{329814}$ & N. fulvodesmus & Kaimon Maru & 600 & 896 & $24.756 \mathrm{~S}$ & $168.104 \mathrm{E}$ & ITS & \\
\hline$G_{329815}$ & N. fulvodesmus & Kaimon Maru & 600 & 896 & $24.756 \mathrm{~S}$ & $168.104 \mathrm{E}$ & ITS & $\mathrm{CO}_{1}$ \\
\hline $\mathrm{G}_{329816}$ & N. zingiberadix & Jumeau East & 470 & 621 & $23.715 \mathrm{~S}$ & $168.257 \mathrm{E}$ & & $\mathrm{CO}_{1}$ \\
\hline $\mathrm{G}_{329817}$ & N. zingiberadix & Jumeau East & 470 & 621 & $23.715 \mathrm{~S}$ & $168.257 \mathrm{E}$ & & $\mathrm{CO}_{1}$ \\
\hline
\end{tabular}


Table 1. Continued

\begin{tabular}{|c|c|c|c|c|c|c|c|c|}
\hline $\begin{array}{l}\text { Queensland Museum } \\
\text { Registration }\end{array}$ & Species & Location & $\begin{array}{l}\text { Depth } \\
\text { Deploy (m) }\end{array}$ & $\begin{array}{l}\text { Depth } \\
\text { Retrieve (m) }\end{array}$ & $\begin{array}{l}\text { Latitude } \\
\text { (Degrees) }\end{array}$ & $\begin{array}{l}\text { Longitude } \\
\text { (Degrees) }\end{array}$ & ITS & $\mathrm{CO}_{1}$ \\
\hline $\mathrm{G}_{329818}$ & N. zingiberadix & Jumeau East & 470 & 621 & $23.715 \mathrm{~S}$ & $168.257 \mathrm{E}$ & & $\mathrm{CO}_{1}$ \\
\hline G329819 & N. zingiberadix & Jumeau East & 470 & 621 & $23.715 \mathrm{~S}$ & $168.257 \mathrm{E}$ & & $\mathrm{CO}_{1}$ \\
\hline $\mathrm{G}_{329821}$ & N. zingiberadix & Jumeau East & 470 & 621 & $23.715 \mathrm{~S}$ & $168.257 \mathrm{E}$ & & $\mathrm{CO}_{1}$ \\
\hline $\mathrm{G}_{329822}$ & N. zingiberadix & Jumeau East & 470 & 621 & $23.715 \mathrm{~S}$ & $168.257 \mathrm{E}$ & & $\mathrm{CO}_{1}$ \\
\hline $\mathrm{G}_{329823}$ & N. zingiberadix & Jumeau East & 470 & 621 & $23.715 \mathrm{~S}$ & $168.257 \mathrm{E}$ & & $\mathrm{CO}_{1}$ \\
\hline $\mathrm{G}_{329824}$ & N. zingiberadix & Jumeau East & 470 & 621 & $23.715 \mathrm{~S}$ & $168.257 \mathrm{E}$ & & $\mathrm{CO}_{1}$ \\
\hline $\mathrm{G}_{329826}$ & N. zingiberadix & Introuvable & 591 & 1032 & $24.663 \mathrm{~S}$ & $168.674 \mathrm{E}$ & & $\mathrm{CO}_{1}$ \\
\hline $\mathrm{G}_{329827}$ & N. zingiberadix & Introuvable & 591 & 1032 & $24.663 \mathrm{~S}$ & $168.674 \mathrm{E}$ & & $\mathrm{CO}_{1}$ \\
\hline $\mathrm{G}_{329828}$ & N. zingiberadix & Introuvable & 591 & 1032 & $24.663 \mathrm{~S}$ & $168.674 \mathrm{E}$ & & $\mathrm{CO}_{1}$ \\
\hline $\mathrm{G}_{329829}$ & N. zingiberadix & Introuvable & 591 & 1032 & $24.663 \mathrm{~S}$ & $168.674 \mathrm{E}$ & & $\mathrm{CO}_{1}$ \\
\hline $\mathrm{G}_{329830}$ & N. zingiberadix & Introuvable & 591 & 1032 & $24.663 \mathrm{~S}$ & $168.674 \mathrm{E}$ & & $\mathrm{CO}_{1}$ \\
\hline $\mathrm{G}_{329831}$ & N. zingiberadix & Introuvable & 591 & 1032 & $24.663 \mathrm{~S}$ & $168.674 \mathrm{E}$ & & $\mathrm{CO}_{1}$ \\
\hline $\mathrm{G}_{329832}$ & N. zingiberadix & Introuvable & 591 & 1032 & $24.663 \mathrm{~S}$ & $168.674 \mathrm{E}$ & & $\mathrm{CO}_{1}$ \\
\hline $\mathrm{G}_{329833}$ & N. zingiberadix & Introuvable & 591 & 1032 & $24.663 \mathrm{~S}$ & $168.674 \mathrm{E}$ & & $\mathrm{CO}_{1}$ \\
\hline $\mathrm{G}_{329834}$ & N. zingiberadix & Introuvable & 591 & 1032 & $24.663 \mathrm{~S}$ & $168.674 \mathrm{E}$ & & $\mathrm{CO}_{1}$ \\
\hline$G_{329835}$ & N. zingiberadix & Introuvable & 591 & 1032 & $24.663 \mathrm{~S}$ & $168.674 \mathrm{E}$ & & $\mathrm{CO}_{1}$ \\
\hline $\mathrm{G}_{318751}$ & I. mirabilis & Jumeau West & 285 & 285 & $23.685 \mathrm{~S}$ & $168.017 \mathrm{E}$ & & $\mathrm{CO}_{1}$ \\
\hline G329906 & I. mirabilis & Jumeau West & 285 & 285 & $23.685 \mathrm{~S}$ & $168.017 \mathrm{E}$ & & $\mathrm{CO}_{1}$ \\
\hline $\mathrm{G}_{329908}$ & I. mirabilis & Jumeau West & 285 & 285 & $23.685 \mathrm{~S}$ & $168.017 \mathrm{E}$ & & $\mathrm{CO}_{1}$ \\
\hline G329909 & I. mirabilis & Jumeau West & 285 & 285 & $23.685 \mathrm{~S}$ & $168.017 \mathrm{E}$ & & $\mathrm{CO}_{1}$ \\
\hline $\mathrm{G}_{329910}$ & I. mirabilis & Jumeau West & 285 & 285 & $23.685 \mathrm{~S}$ & $168.017 \mathrm{E}$ & & $\mathrm{CO}_{1}$ \\
\hline $\mathrm{G}_{329911}$ & I. mirabilis & Jumeau West & 285 & 285 & $23.685 \mathrm{~S}$ & $168.017 \mathrm{E}$ & & $\mathrm{CO}_{1}$ \\
\hline G329912 & I. mirabilis & Jumeau West & 285 & 285 & $23.685 \mathrm{~S}$ & $168.017 \mathrm{E}$ & & $\mathrm{CO}_{1}$ \\
\hline $\mathrm{G}_{329913}$ & I. mirabilis & Jumeau West & 285 & 285 & $23.685 \mathrm{~S}$ & $168.017 \mathrm{E}$ & & $\mathrm{CO}_{1}$ \\
\hline G329914 & I. mirabilis & Jumeau West & 285 & 285 & $23.685 \mathrm{~S}$ & $168.017 \mathrm{E}$ & & $\mathrm{CO}_{1}$ \\
\hline $\mathrm{G}_{329915}$ & I. mirabilis & Jumeau West & 285 & 285 & $23.685 \mathrm{~S}$ & $168.017 \mathrm{E}$ & & $\mathrm{CO}_{1}$ \\
\hline $\mathrm{G}_{329916}$ & I. mirabilis & Kaimon Maru & 320 & 345 & $24.753 \mathrm{~S}$ & $168.121 \mathrm{E}$ & & $\mathrm{CO}_{1}$ \\
\hline $\mathrm{G}_{329917}$ & I. mirabilis & Kaimon Maru & 320 & 345 & $24.753 \mathrm{~S}$ & $168.121 \mathrm{E}$ & & $\mathrm{CO}_{1}$ \\
\hline $\mathrm{G}_{329918}$ & I. mirabilis & Kaimon Maru & 320 & 345 & $24.753 \mathrm{~S}$ & $168.121 \mathrm{E}$ & & $\mathrm{CO}_{1}$ \\
\hline $\mathrm{G}_{329919}$ & I. mirabilis & Kaimon Maru & 320 & 345 & $24.753 \mathrm{~S}$ & $168.121 \mathrm{E}$ & & $\mathrm{CO}_{1}$ \\
\hline $\mathrm{G}_{329920}$ & I. mirabilis & Kaimon Maru & 320 & 345 & $24.753 \mathrm{~S}$ & $168.121 \mathrm{E}$ & & $\mathrm{CO}_{1}$ \\
\hline G329921 & I. mirabilis & Kaimon Maru & 320 & 345 & $24.753 \mathrm{~S}$ & $168.121 \mathrm{E}$ & & $\mathrm{CO}_{1}$ \\
\hline $\mathrm{G}_{329922}$ & I. mirabilis & Kaimon Maru & 320 & 345 & $24.753 \mathrm{~S}$ & $168.121 \mathrm{E}$ & & $\mathrm{CO}_{1}$ \\
\hline $\mathrm{G}_{329923}$ & I. mirabilis & Kaimon Maru & 320 & 345 & $24.753 \mathrm{~S}$ & $168.121 \mathrm{E}$ & & $\mathrm{CO}_{1}$ \\
\hline G329924 & I. mirabilis & Kaimon Maru & 320 & 345 & $24.753 \mathrm{~S}$ & $168.121 \mathrm{E}$ & & $\mathrm{CO}_{1}$ \\
\hline G329925 & I. mirabilis & Kaimon Maru & 320 & 345 & $24.753 \mathrm{~S}$ & $168.121 \mathrm{E}$ & & $\mathrm{CO}_{1}$ \\
\hline $\mathrm{G}_{329926}$ & I. mirabilis & Blanc Nouveau 2 & 275 & 348 & $23.277 \mathrm{~S}$ & $168.233 \mathrm{E}$ & & $\mathrm{CO}_{1}$ \\
\hline $\mathrm{G}_{329927}$ & I. mirabilis & Blanc Nouveau 2 & 275 & 348 & $23.277 \mathrm{~S}$ & $168.233 \mathrm{E}$ & & $\mathrm{CO}_{1}$ \\
\hline G329929 & I. mirabilis & Blanc Nouveau 2 & 275 & 348 & $23.277 \mathrm{~S}$ & $168.233 \mathrm{E}$ & & $\mathrm{CO}_{1}$ \\
\hline $\mathrm{G}_{329930}$ & I. mirabilis & Blanc Nouveau 2 & 275 & 348 & $23.277 \mathrm{~S}$ & $168.233 \mathrm{E}$ & & $\mathrm{CO}_{1}$ \\
\hline $\mathrm{G}_{329931}$ & I. mirabilis & Blanc Nouveau 2 & 275 & 348 & $23.277 \mathrm{~S}$ & $168.233 \mathrm{E}$ & & $\mathrm{CO}_{1}$ \\
\hline G329932 & I. mirabilis & Blanc Nouveau 2 & 275 & 348 & $23.277 \mathrm{~S}$ & $168.233 \mathrm{E}$ & & $\mathrm{CO}_{1}$ \\
\hline $\mathrm{G}_{329933}$ & I. mirabilis & Blanc Nouveau 2 & 275 & 348 & $23.277 \mathrm{~S}$ & $168.233 \mathrm{E}$ & & $\mathrm{CO}_{1}$ \\
\hline $\mathrm{G}_{329934}$ & I. mirabilis & Blanc Nouveau 2 & 275 & 348 & $23.277 \mathrm{~S}$ & $168.233 \mathrm{E}$ & & $\mathrm{CO}_{1}$ \\
\hline G329935 & I. mirabilis & Blanc Nouveau 2 & 275 & 348 & $23.277 \mathrm{~S}$ & $168.233 \mathrm{E}$ & & $\mathrm{CO}_{1}$ \\
\hline $\mathrm{G}_{329936}$ & I. mirabilis & Munida & 270 & 350 & $22.998 \mathrm{~S}$ & $168.363 \mathrm{E}$ & & $\mathrm{CO}_{1}$ \\
\hline $\mathrm{G}_{329937}$ & I. mirabilis & Munida & 270 & 350 & $22.998 \mathrm{~S}$ & $168.363 \mathrm{E}$ & & $\mathrm{CO}_{1}$ \\
\hline $\mathrm{G}_{329938}$ & I. mirabilis & Munida & 270 & 350 & $22.998 \mathrm{~S}$ & $168.363 \mathrm{E}$ & & $\mathrm{CO}_{1}$ \\
\hline G329939 & I. mirabilis & Munida & 270 & 350 & $22.998 \mathrm{~S}$ & $168.363 \mathrm{E}$ & & $\mathrm{CO}_{1}$ \\
\hline G329940 & I. mirabilis & Munida & 270 & 350 & $22.998 \mathrm{~S}$ & $168.363 \mathrm{E}$ & & $\mathrm{CO}_{1}$ \\
\hline $\mathrm{G}_{329941}$ & I. mirabilis & Munida & 270 & 350 & $22.998 \mathrm{~S}$ & $168.363 \mathrm{E}$ & & $\mathrm{CO}_{1}$ \\
\hline $\mathrm{G}_{329942}$ & I. mirabilis & Munida & 270 & 350 & $22.998 \mathrm{~S}$ & $168.363 \mathrm{E}$ & & $\mathrm{CO}_{1}$ \\
\hline G329943 & I. mirabilis & Munida & 270 & 350 & $22.998 \mathrm{~S}$ & $168.363 \mathrm{E}$ & & $\mathrm{CO}_{1}$ \\
\hline G329944 & I. mirabilis & Munida & 270 & 350 & $22.998 \mathrm{~S}$ & $168.363 \mathrm{E}$ & & $\mathrm{CO}_{1}$ \\
\hline $\mathrm{G}_{329945}$ & I. mirabilis & Munida & 270 & 350 & $22.998 \mathrm{~S}$ & $168.363 \mathrm{E}$ & & $\mathrm{CO}_{1}$ \\
\hline$G_{329952}$ & N. zingiberadix & Jumeau East & 470 & 621 & $23.715 \mathrm{~S}$ & $168.257 \mathrm{E}$ & & $\mathrm{CO}_{1}$ \\
\hline G329953 & N. zingiberadix & Introuvable & 591 & 1032 & $24.663 \mathrm{~S}$ & $168.674 \mathrm{E}$ & & $\mathrm{CO}_{1}$ \\
\hline $\mathrm{G}_{331823}$ & N. zingiberadix & Introuvable & 591 & 1032 & $24.663 \mathrm{~S}$ & $168.674 \mathrm{E}$ & & $\mathrm{CO}_{1}$ \\
\hline $\mathrm{G}_{331825}$ & N. zingiberadix & Introuvable & 591 & 1032 & $24.663 \mathrm{~S}$ & $168.674 \mathrm{E}$ & & $\mathrm{CO}_{1}$ \\
\hline $\mathrm{G}_{331827}$ & N. zingiberadix & Introuvable & 591 & 1032 & $24.663 \mathrm{~S}$ & $168.674 \mathrm{E}$ & & $\mathrm{CO}_{1}$ \\
\hline G331828 & N. zingiberadix & Introuvable & 591 & 1032 & $24.663 \mathrm{~S}$ & $168.674 \mathrm{E}$ & & $\mathrm{CO}_{1}$ \\
\hline G331829 & N. zingiberadix & Introuvable & 591 & 1032 & $24.663 \mathrm{~S}$ & $168.674 \mathrm{E}$ & & $\mathrm{CO}_{1}$ \\
\hline $\mathrm{G}_{331830}$ & N. zingiberadix & Introuvable & 591 & 1032 & $24.663 \mathrm{~S}$ & $168.674 \mathrm{E}$ & & $\mathrm{CO}_{1}$ \\
\hline
\end{tabular}


Table 1. Continued

\begin{tabular}{|c|c|c|c|c|c|c|c|c|}
\hline $\begin{array}{l}\text { Queensland Museum } \\
\text { Registration }\end{array}$ & Species & Location & $\begin{array}{l}\text { Depth } \\
\text { Deploy (m) }\end{array}$ & $\begin{array}{l}\text { Depth } \\
\text { Retrieve (m) }\end{array}$ & $\begin{array}{l}\text { Latitude } \\
\text { (Degrees) }\end{array}$ & $\begin{array}{l}\text { Longitude } \\
\text { (Degrees) }\end{array}$ & ITS & $\mathrm{CO}_{1}$ \\
\hline $\mathrm{G}_{331831}$ & N. zingiberadix & Introuvable & 591 & 1032 & $24.663 \mathrm{~S}$ & $168.674 \mathrm{E}$ & & $\mathrm{CO}_{1}$ \\
\hline $\mathrm{G}_{331832}$ & N. zingiberadix & Jumeau East & 470 & 621 & $23.715 \mathrm{~S}$ & $168.257 \mathrm{E}$ & & $\mathrm{CO}_{1}$ \\
\hline $\mathrm{G}_{331833}$ & N. zingiberadix & Jumeau East & 470 & 621 & $23.715 \mathrm{~S}$ & $168.257 \mathrm{E}$ & & $\mathrm{CO}_{1}$ \\
\hline $\mathrm{G}_{331834}$ & N. zingiberadix & Jumeau East & 470 & 621 & $23.715 \mathrm{~S}$ & $168.257 \mathrm{E}$ & & $\mathrm{CO}_{1}$ \\
\hline $\mathrm{G}_{331836}$ & N. zingiberadix & Jumeau East & 470 & 621 & $23.715 \mathrm{~S}$ & $168.257 \mathrm{E}$ & & $\mathrm{CO}_{1}$ \\
\hline$G_{331837}$ & N. zingiberadix & Jumeau East & 470 & 621 & $23.715 \mathrm{~S}$ & $168.257 \mathrm{E}$ & & $\mathrm{CO}_{1}$ \\
\hline
\end{tabular}

(Edgar, 2004). Sequences are published in NCBI GenBank (Acc. No. KR270646-KR270725) and in the Sponge Barcoding Database (www.spongebarcoding.org).

\section{Analyses of genetic variation}

Genetic diversity was expressed as nucleotide diversity $(\pi$; the probability that two randomly chosen homologous nucleotides differ) and gene diversity ( $h$; the probability that two randomly chosen genotypes differ), and calculated in Arlequin 3.1 (Excoffier et al., 2005). The hierarchical distribution of genetic diversity was analysed in the AMOVA framework (Analysis of Molecular Variance) also using Arlequin 3.1, where genetic variation was partitioned into withinindividual, among-individual within-population, and amongpopulation components. Significance of variance components was assessed using 10 ooo randomizations. Within-individual variation was included as a variance component due to the presence of IGPs. Pairwise $F_{\mathrm{ST}}$ s between sampled populations were calculated in Arlequin 3.1. To indirectly assess the contribution of sexual $v s$ asexual modes of reproduction, we examined levels of linkage disequilibrium, where high levels indicate either non-random sexual reproduction (inbreeding) or asexual reproduction. Linkage disequilibrium was calculated in Arlequin 3.1 (Excoffier et al., 2005). Analyses of the three lithistid species were carried out independently. Sequencing of the ITS was only carried out for N. fulvodesmus. The CO1 fragment of $N$. fulvodesmus was only sequenced on eight individuals to detect if there was any variation within this species, while all collected I. mirabilis and N. zingiberadix were screened.

\section{Phylogenetic analyses}

Maximum likelihood reconstructions were performed with RAxML-7.2.5 (Stamatakis, 2006) under the GTR + GAMMA model of substitution. Branch support was inferred with 1000 rapid bootstrap replicates.

\section{RESULTS}

\section{Neoschrammeniella fulvodesmus}

A total of 114 ITS genotypes were recovered from the 57 specimens (Table 2). A higher number of genotypes, and higher gene and nucleotide diversities were found for ITS 1 than for $\mathrm{ITS}_{2}$ and $5.8 \mathrm{~S}$. There were 24 nucleotide differences in the 5.8S rRNA section, with only six single base changes found in the $5.8 \mathrm{~S}$ region. One sequence had two of these changes and only two sequences shared the same change and they were from different seamounts. Overall gene diversity was high $(h=0.9843)$ when compared with nucleotide diversity $(\pi=0.127324)$.

Six of the 114 genotypes were found in a single specimen. In two of the 57 specimens, only one plasmid was successfully sequenced. Of the remaining 55 individuals for which multiple sequences were obtained, 52 (94.5\%) exhibited intra-genomic polymorphism, with between two and six genotypes present within an individual specimen.

An AMOVA looking at the individuals and the variation within each individual shows $84.57 \%$ of the variation is within the individuals, compared with $15.66 \%$ between the individuals within each seamount (Table 3). The most common genotype occurred 14 times and 63 genotypes occurred only once.

The AMOVA for all of the populations and for the entire sequence including ITS 1 and ITS 2 indicates that virtually all of the variation $(97.38 \%)$ is contained within each seamount (Table 4).

Most of the genotypes $(71 / 114)$ were found on a single seamount, with the number of unique genotypes ranging from six at Zorro North to 19 at Eponge South (Figure 1). Most shared genotypes were limited to two seamounts (40 genotypes), however, one genotype occurred at three seamounts (Jumeau East, Eponge North and Zorro North). The most common genotype (14 specimens) occurred at four seamounts (Jumeau East, Kaimon Maru, Eponge South and Zorro North)

Table 2. Comparison of diversities and $F_{\mathrm{ST}} \mathrm{S}$ of $N$. fulvodesmus calculated from ITS on the seamounts in order of increasing latitude.

\begin{tabular}{|c|c|c|c|c|c|c|}
\hline Seamount & Sample size (N) & $\begin{array}{l}\text { Number of } \\
\text { sequences }\end{array}$ & $\begin{array}{l}\text { Number of } \\
\text { genotypes }(h)\end{array}$ & $\begin{array}{l}\text { Nucleotide } \\
\text { diversity }(\pi)\end{array}$ & $\begin{array}{l}\text { Gene } \\
\text { diversity }(h)\end{array}$ & $F_{\mathrm{ST}}$ \\
\hline Jumeau East & 9 & 31 & 20 & 0.101965 & 0.9600 & 0.04672 \\
\hline Kaimon-Maru & 9 & 39 & 35 & 0.248202 & 0.9638 & 0.01040 \\
\hline Eponge North & 10 & 23 & 21 & 0.311829 & 1.0000 & -0.00279 \\
\hline Eponge South & 10 & 35 & 29 & 0.046155 & 0.8758 & 0.06069 \\
\hline Zorro North & 9 & 31 & 27 & 0.315330 & 0.9815 & -0.00678 \\
\hline Zorro South & 10 & 38 & 29 & 0.100148 & 0.9644 & 0.04725 \\
\hline All & 57 & 197 & 152 & 0.127324 & 0.9843 & \\
\hline
\end{tabular}


Table 3. AMOVA results of the entire ITS sequence of N. fulvodesmus between seamounts, between individuals within seamounts and within individuals.

\begin{tabular}{lcccc}
\hline Source of variation & Degrees of freedom & Sum of squares & Variance components & Percentage of variation \\
\hline Between seamounts & 5 & 625.243 & -0.22734 & -0.24 \\
Between individual genotypes & 43 & 5052.946 & 14.94180 & 0.15426 \\
$\quad$ within seamounts & 77 & & & 15.66 \\
Within individuals & 125 & 6211.692 & 80.67132 & 84.57 \\
Total & & $11,889.881$ & 95.38578 & \\
\hline
\end{tabular}

and the second most common genotype (8 specimens) also occurred at four seamounts (Jumeau East, Kaimon Maru, Eponge South and Zorro South). No genotype was shared across all six seamounts.

When each seamount is regarded as a distinct population, the pairwise genetic differences (Table 5) showed that the two pairs of populations that are physically closest together, i.e. Zorro North and Zorro South, and Eponge North and Eponge South, show significant pairwise $F_{\mathrm{ST}}$. The only other significant pairwise differences are between Zorro South and Jumeau East, and between Zorro South and Eponge South.
When the seamounts are clustered geographically, the variation is distributed within each population rather than between populations or groups of populations even when adjacent seamounts are compared with other seamounts. There is no latitudinal gradient showing genetic flow from the north to south nor is there flow from the south to the north. There was no latitudinal gradient between the seamounts. Neither was there any longitudinal gradient for gene diversity, nucleotide diversity and haplotypic diversity between the seamounts. There were no significant bathymetric gradients, or bathymetric ranges amongst the genotypes or the

Table 4. AMOVA results of the entire ITS sequence of $N$. fulvodesmus between seamounts and within seamounts.

\begin{tabular}{lcccc}
\hline Source of variation & Degrees of freedom & Sum of squares & Variance components & Percentage of variation \\
\hline Between seamounts & 5 & 592.141 & 2.02720 & 2.62 \\
Within seamounts & 123 & 9254.269 & 75.23796 & 97.38 \\
Total & 128 & 9846.411 & 77.26516 & \\
\hline
\end{tabular}

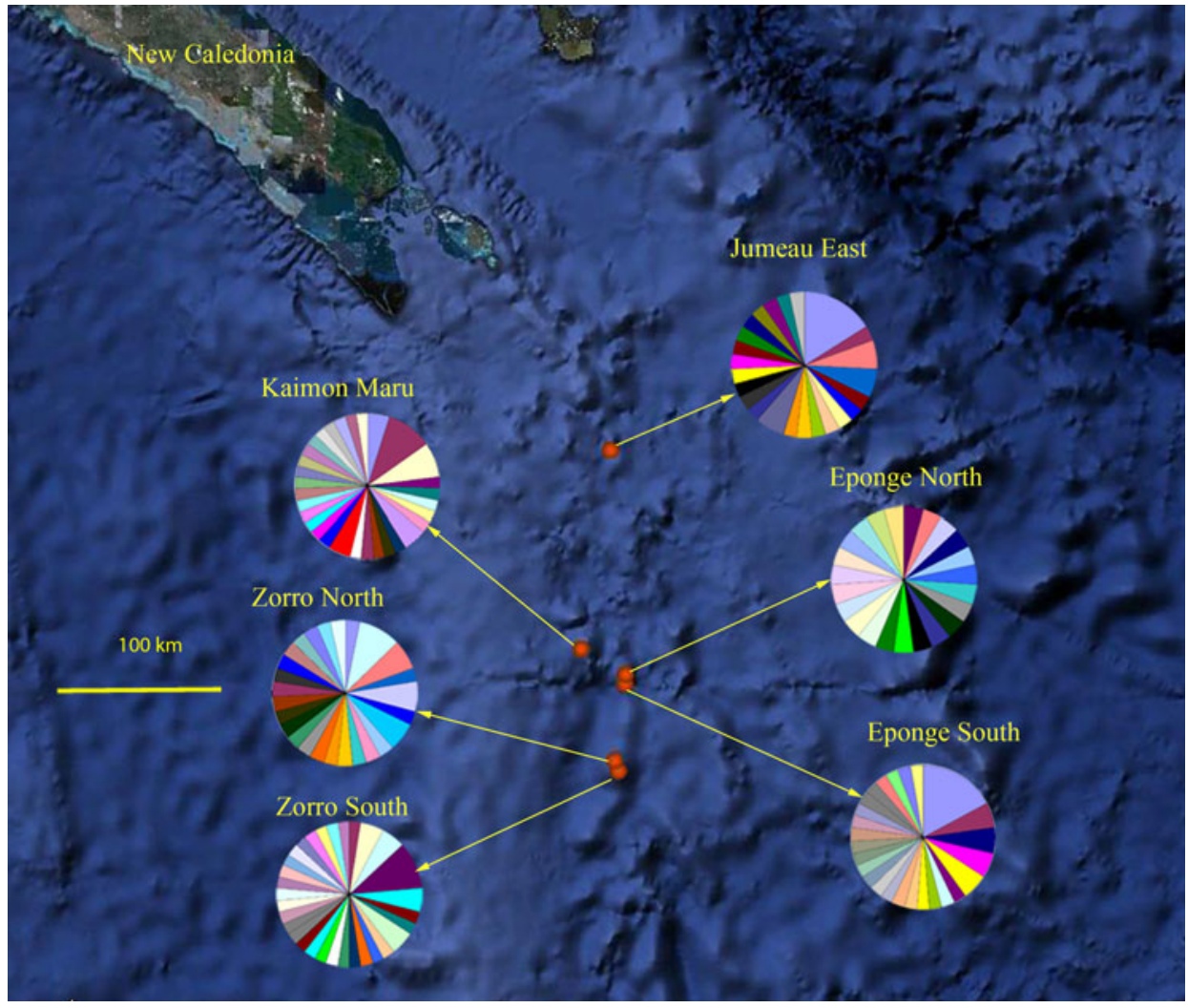

Fig. 1. Distribution of the N. fulvodesmus ITS genotypes on the seamounts (ALA, 2014). 
Table 5. Pairwise differences in $F_{\mathrm{ST}}$ of $N$. fulvodesmus calculated from ITS between the seamounts using the distance method.

\begin{tabular}{lccccc}
\hline Seamount & Jumeau East & Kaimon-Maru & Eponge North & Eponge South & Zorro North \\
\hline Jumeau East & 0.00000 & & & & \\
Kaimon-Maru & 0.01597 & 0.00000 & 0.00000 & & \\
Eponge North & 0.06331 & -0.02924 & $0.11365^{*}$ & 0.00000 & 0.00000 \\
Eponge South & -0.01924 & 0.05118 & 0.06110 & 0.00663 & $0.06125^{*}$ \\
Zorro North & -0.02223 & 0.01823 & -0.03920 & $0.09611^{*}$ & 0.00000 \\
Zorro South & $0.06132^{*}$ & -0.02529 & & & 0.000 \\
\hline
\end{tabular}

*Indicates significant difference from 0 at $P=0.05$.

diversities. Most of the depths of the dredges on the seamounts from which the specimens of $N$. fulvodesmus were recovered were from around $500 \mathrm{~m}$ depth (ranging from 470 to $691 \mathrm{~m}$ ) (Table 1), with the exception of Kaimon Maru and Zorro North which reached depths of 896 and $1000 \mathrm{~m}$ respectively. Interestingly the highest diversities were found from the Eponge North seamount with the smallest depth range of dredges.

All of the individual populations showed significant levels of linkage disequilibrium amongst the polymorphic loci as shown in Table 6.

Eight individuals were screened using $\mathrm{CO}_{1}$ from three seamounts to detect if there was any variation within this species. Table 7 compares the diversity of the seamounts, which is zero, and there were no polymorphic loci found in the $\mathrm{CO}_{1}$ fragment. All eight individuals were found to belong to the same mtDNA genotype and were found at all three seamounts (Figure 3). These eight individuals when analysed with ITS found 23 of the 114 genotypes. This ranged from two individuals with only one genotype detected to another individual with eight genotypes. These are likely to be related to the number of clones sequenced.

Pairwise differences in $F_{\mathrm{ST}}$ using the distance method between the seamounts for CO1was not calculated for N. fulvodesmus because of the low numbers of individuals sampled from the population, likewise linkage disequilibrium could not be calculated because there were no polymorphic loci. All the loci at the $\mathrm{CO}_{1}$ fragment were monomorphic. No morphological differences were detected between the specimens.

\section{Neoaulaxinia zingiberadix}

All 32 specimens of $N$. zingiberadix were identical for $\mathrm{CO}_{1}$ sequenced region (Figure 3). There was no latitudinal,

Table 6. Summary of significant polymorphic loci of $N$. fulvodesmus with linkage disequilibrium.

\begin{tabular}{llll}
\hline Seamount & $\begin{array}{l}\text { Number of } \\
\text { combinations }\end{array}$ & $\begin{array}{l}\text { Loci significant } \\
\text { disequilibrium } \\
(\boldsymbol{P}=\text { o.05) }\end{array}$ & $\begin{array}{l}\text { \% of loci with } \\
\text { significant linkage } \\
\text { disequilibrium }\end{array}$ \\
\hline Jumeau East & 171 & 28 & $16.4 \%$ \\
Zorro South & 435 & 50 & $11.5 \%$ \\
Zorro North & 105 & 20 & $19.0 \%$ \\
Eponge North & 231 & 13 & $5.6 \%$ \\
Eponge South & 276 & 35 & $12.7 \%$ \\
Kaimon-Maru & 435 & 63 & $14.5 \%$ \\
Combined & 45 & 26 & $57.8 \%$ \\
\multicolumn{1}{c}{ populations } & & & \\
\hline
\end{tabular}

longitudinal or depth separation. All specimens were from two seamounts Jumeau East and Introvable and there was no separation between the seamounts (Table 8).

Linkage disequilibrium was not calculated because of the monomorphism at all the loci of the $\mathrm{CO}_{1}$ fragment. No morphological differences were detected between the specimens.

\section{Isabella mirabilis}

Sequencing of the $\mathrm{CO}_{1}$ fragment of all specimens of the species I. mirabilis, divided the specimens into four distinct genotypes with one very large genotype comprising the majority of specimens $(23 / 38)$ (Figure 2). These sponges were from four seamount sites, each seamount with three or four genotypes (Table 9). There was one genotype present only in two specimens. There were other genotypes comprised of five and eight individuals (Figure 3). All four seamounts had three of the four genotypes in different combinations, so there was no specialization of genotypes for a specific seamount. All of the specimens were collected from between 270 and $348 \mathrm{~m}$ depth (Table 1) and the genotypes were not specific for any particular depth. Morphological analysis of the genotypes of I. mirabilis only revealed variation in the relative proportions of microscleres.

Significant differences were found in $F_{\mathrm{ST}}$ values in seamounts of the species I. mirabilis between Jumeau West and Munida, and Jumeau West and Blanc Nouveau 2 only (Table 10, see also Figure 2). In order to test for population subdivision the seamounts were clustered into two groups as identified by $F_{\mathrm{ST}}$ analysis. One group contained the two seamounts of Munida and Blanc Nouveau 2, and the other group contained the seamounts Jumeau West and Kaimon Maru. This revealed that $97.79 \%$ of the variation was found within the seamount clusters, so there was no population structure here. Clustering of the seamounts using a distance-related latitudinal gradient provided a higher signal of the variation spread between the populations, with clustering of Jumeau West, Munida and Blanc Nouveau 2 compared with Kaimon Maru. On all accounts most of the variation is included within the populations (92.66\%).

Linkage disequilibrium was calculated for the polymorphic loci at the seamounts. For two sites, Munida and Blanc Nouveau 2, there were no polymorphic loci with significant linkage disequilibrium. This contrasts with the other two seamounts of Jumeau West and Kaimon Maru, which had both of the polymorphic loci showing significant $(P=0.05)$ linkage disequilibrium. This is all overshadowed by the fact that for the $\mathrm{CO}_{1}$ region from the 597 usable nucleotide sites there were polymorphisms only at a maximum of three sites for Munida and all other seamounts had two polymorphic 
Table 7. Comparison of diversities of the seamounts of N. fulvodesmus from $\mathrm{CO}_{1}$ in order of increasing latitude.

\begin{tabular}{llllll}
\hline Seamount & Sample size $(\mathbf{N})$ & Number of sequences & Number of genotypes $(\boldsymbol{h})$ & Nucleotide diversity $(\boldsymbol{\pi})$ & Gene diversity $(\boldsymbol{h})$ \\
\hline Jumeau East & 4 & 4 & 1 & 0 & 1 \\
Kaimon Maru & 3 & 3 & 1 & 0 & 1 \\
Zorro South & 1 & 1 & 1 & 0 & 1 \\
Total & 8 & 8 & 1 & 0 & 1 \\
\hline
\end{tabular}

Table 8. Comparison of diversities of N. zingiberadix calculated from $\mathrm{CO}_{1}$ of the seamounts in order of increasing latitude.

\begin{tabular}{llllll}
\hline Seamount & Sample size $(\mathbf{N})$ & Number of sequences & Number of genotypes $(\boldsymbol{h})$ & Nucleotide diversity $(\boldsymbol{\pi})$ & Gene diversity $(\boldsymbol{h})$ \\
\hline Jumeau East & 14 & 14 & 1 & 0 & 1 \\
Introuvable & 18 & 18 & 1 & 0 & 0 \\
Total & 32 & 32 & 1 & 0 & 1 \\
\hline
\end{tabular}

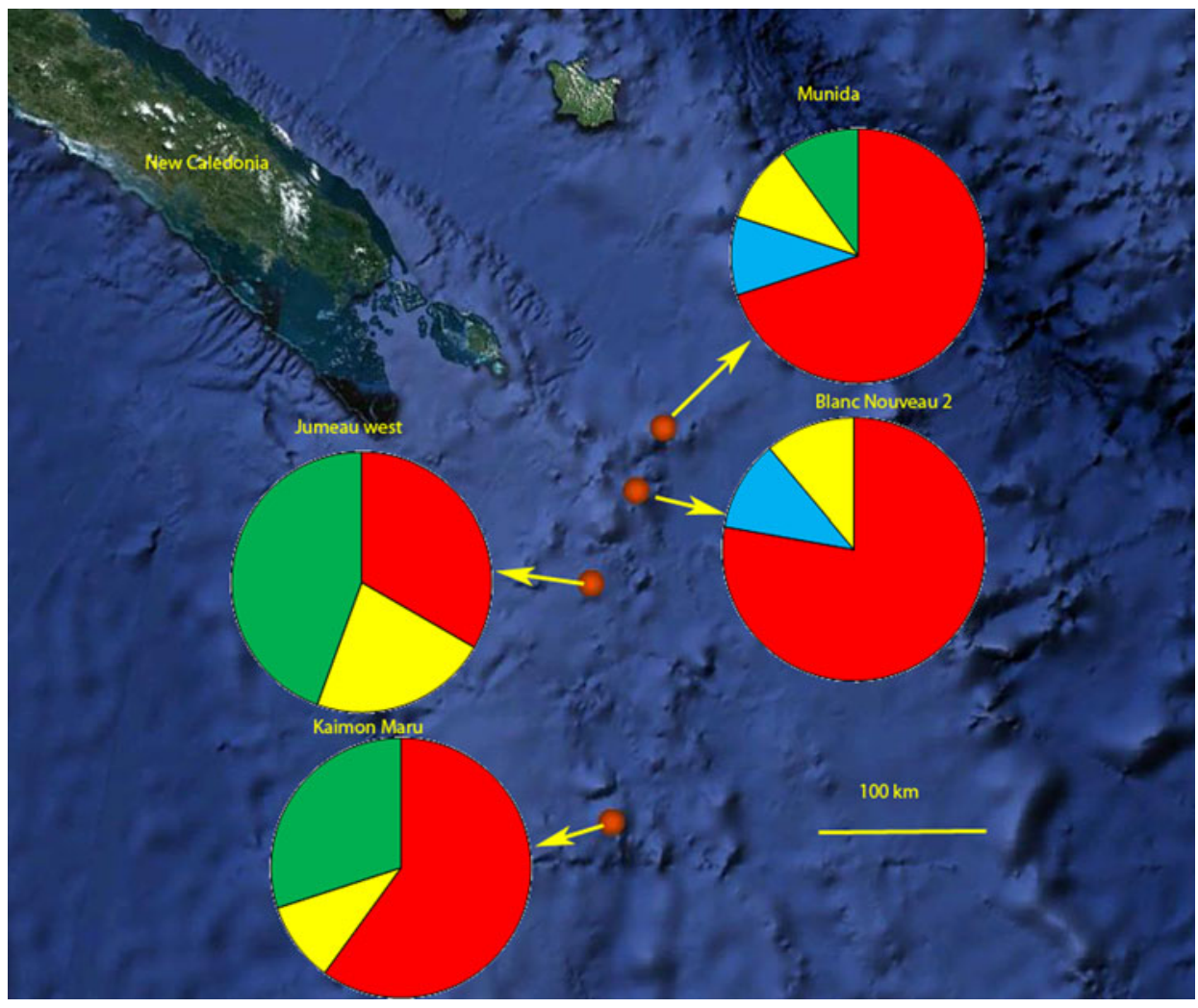

Fig. 2. Distribution of the I. mirabilis CO1 genotypes on the seamounts (ALA, 2014).

Table 9. Comparison of diversities of I. mirabilis calculated from $\mathrm{CO}_{1}$ of the seamounts in order of increasing latitude.

\begin{tabular}{lrclcc}
\hline Seamount & Sample size $(\mathbf{N})$ & Number of sequences & Number of genotypes $(\boldsymbol{h})$ & Nucleotide diversity $(\boldsymbol{\pi})$ & Gene diversity $(\boldsymbol{h})$ \\
\hline Munida & 10 & 10 & 4 & 0.001266 & 0.000723 \\
Blanc Nouveau 2 & 9 & 9 & 3 & 0.001719 & 1 \\
Jumeau WestWest & 9 & 9 & 3 & 0.002049 & 1 \\
Kaimon Maru & 10 & 10 & 4 & 0.010 & 1 \\
Total & 38 & 38 & 3 & 1 \\
\hline
\end{tabular}




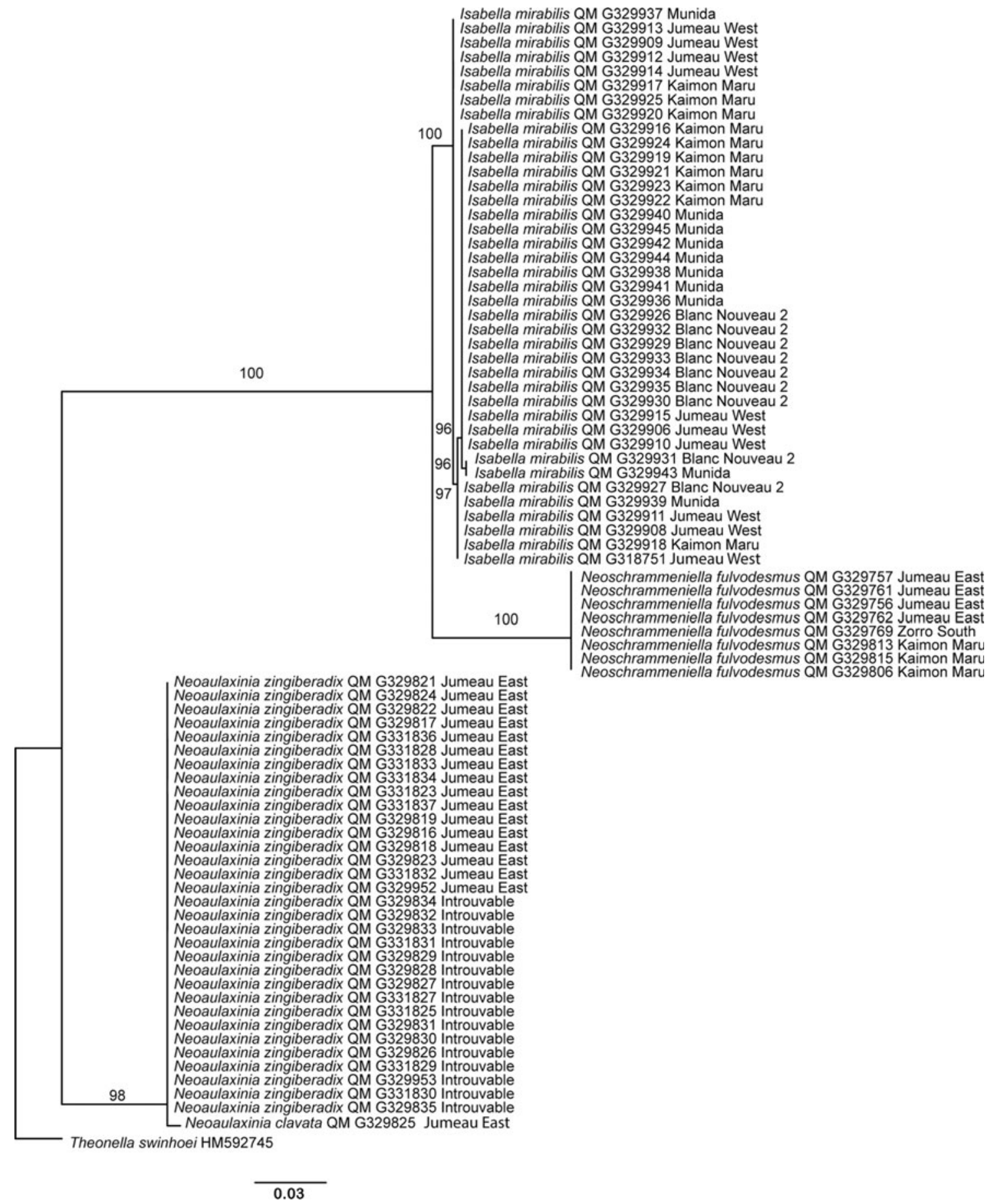

Fig. 3. CO1 maximum likelihood reconstruction of the samples analysed in this study. Numbers following the taxon names are Queensland Museum collection numbers (QM G) or GenBank accession numbers. Numbers on the branches indicate rapid bootstrap support values ( $>70)$. The scale bar depicts substitutions per site.

sites. This contrasts strongly to the two other species, which showed no polymorphism at all for the CO1.

\section{DISCUSSION}

This study demonstrates that the three lithistid sponge populations have varying levels of population connectivity amongst seamounts, ranging from very well connected subpopulations on individual seamounts to subpopulations with moderate gene flows between the seamounts. Seamounts are topographically small and isolated, suggesting an island model, which has led to an expectation of high endemism and species richness on seamounts (Richer de Forges et al., 2000). From a purely geomorphological sense one would expect each separate seamount to behave at least as a discrete population. These seamounts would then have reduced gene flow and be particularly susceptible to genetic drift because of their small size (Le Goff-Vitry et al., 2004). However, the results in the present study did not support this. Contrary to the island 
Table 1o. Pairwise differences in $F_{\mathrm{ST}}$ of I. mirabilis calculated from $\mathrm{CO}_{1}$ between the seamounts using the distance method.

\begin{tabular}{lcclc}
\hline Seamount & Munida & $\begin{array}{l}\text { Blanc } \\
\text { Nouveau 2 }\end{array}$ & $\begin{array}{l}\text { Jumeau } \\
\text { West }\end{array}$ & $\begin{array}{l}\text { Kaimon } \\
\text { Maru }\end{array}$ \\
\hline Munida & 0.00000 & & & \\
Blanc Nouveau 2 & -0.08221 & 0.00000 & & \\
Jumeau West & $0.21921^{*}$ & $0.36719^{*}$ & 0.00000 & \\
Kaimon Maru & -0.00076 & -0.00130 & -0.01170 & 0.00000 \\
\hline
\end{tabular}

*Indicates significant difference from 0 at $P=0.05$.

model, seamounts are not surrounded by hydrological barriers and populations might have high gene flow between seamounts, a finding also concluded by other researchers (Samadi et al., 2007; Clark et al., 2012). The lack of significant difference between the sites studied here indicates all the seamounts form one meta-population. The alleged high rates of gene flow of the species studied here amongst the seamounts are presumably caused largely by currents, e.g. migration of larvae, sponge tissue drifting or transmission on ghost nets. With potentially year-round reproduction sponges would be able to take advantage of differing seasonal currents to migrate (Longo et al., 2012). The concept of a short-lived crawling stage as suggested for some sponge species (Hooper \& Van Soest, 2002) is likely to be untenable here. Indeed, the presence of the large trough between these seamounts likely represents a serious barrier to crawling larvae. It is most likely that the sponges studied here have a highly mobile longer-lived egg and sperm stage or egg/zygote phase than previously thought which enables them to survive the distance between the seamounts. Other studies have found that species of molluscs with high genetic structure have larvae with low dispersal ability and species with low genetic structure have larvae with high dispersal ability (Todd et al., 1998; Boisselier-Dubayle, 1999; Kyle \& Boulding, 2000; Collin, 2001). Bohonak (1999) indicated there is a correspondence between dispersal and gene flow.

All specimens of Neoaulaxinia zingiberadix and Neoschrammeniella fulvodesmus, when sequenced for CO1, showed only one single genotype. CO1 sequencing of Isabella mirabilis revealed four genotypes and each seamount had three of the four genotypes. There was no specialization for genotypes on each seamount. Therefore this species is an example of a lithistid species that mixes well between seamounts. This also confirms that $\mathrm{CO}_{1}$ provides resolution at population level for this species. Other researchers using $\mathrm{CO}_{1}$ have also reported low numbers of genotypes, although in different sponge species (Wörheide, 2006; Hoshino et al., 2008; Whalan et al., 2008; Dailianis et al., 2011). The presence of only one genotype in $N$. zingiberadix and $N$. fulvodesmus hints that genetic drift has occurred. The formation of external buds of hexactinellids in the stable environment of the Antarctic waters (Teixido et al., 2006) may enable the formation of a stable clonal population in the immediate vicinity. Indeed the formation of buds was observed on one of the specimens of I. mirabilis in this study. If the ancestral population of one of these lithistid sponges was separated a long time ago but reproduced clonally we would expect two discrete populations. All the seamounts are most likely functioning as a single population suggested by the presence of one single $\mathrm{CO}_{1}$ genotype of $N$. zingiberadix and N. fulvodesmus. An alternative explanation to the lack of genotypic diversity in $\mathrm{CO}_{1}$ is because the rock sponges are long lived and slow growing coupled with slow mitochondrial substitutions (Shearer et al., 2002). The overwhelming proportion of diversity detected by ITS within the same specimens of $N$. fulvodesmus is contained within intragenomic diversity, and in comparison there are comparatively small differences within seamounts and virtually no differences between seamounts. With the ITS there were no examples of the same genotype occurring at all six sites, but there were many unique genotypes at each seamount. Because these species appear to be very slow growing and long lived there does not need to be much gene flow between the seamounts to maintain the genetic uniformity and the vagility of the larval stage may still be quite low. In theory, the exchange of a single individual between large populations is enough to counter any effects from genetic drift (Silva \& Russo, 2000) and with a sponge with a generation time of potentially several hundred years this is not impossible.

Clusters of ITS genotypes in N. fulvodesmus on seamounts are likely to be the result of either: adjacent settling of larvae, clonality via asexual reproduction, colony fragmentation into discrete individuals, inbreeding populations or even selffertilizing populations. However, it could also be a function of sampling as deep sea lithistids have been observed to adhere to the rock in distinct clusters (Ekins' personal observations from ROV research Deep Down Under Expedition; www. deepdownunder.de). Much of the diversity in sponge populations has previously been attributed to sex and not asexuality (Dailianis et al., 2011; Uriz \& Turon, 2012). However, asexual components may also add to the diversity by colony formation of multiple genotypes such as chimeras. Chimeras have been reported in other sessile marine invertebrates, e.g. corals (Puill-Stephan et al., 2009), ascidians (Sommerfeldt et al., 2003) as well as sponges (Wulff, 1990; Maldonado, 1998; Blanquer \& Uriz, 2011). Somatic mutations have also been suggested as a potential mean for increasing genotypic diversity within an individual (Buss, 1982), but these options appear less likely to explain the diversity observed here.

A sexual population is evidenced by the variation in the ITS region in N. fulvodesmus, high levels of gene and genotypic diversity coupled with low levels of nucleotide diversity. However, while within this species there are also indirect indicators of asexual components as evidenced by the occurrence of the same common genotypes on the different seamounts and significant linkage disequilibrium, however asexual reproduction in rock sponges has yet to be described. For N. fulvodesmus and N. zingiberadix the dominance of one genotype in CO1 may be seen as an indication of asexuality, however this has to be tempered by the conservation of the $\mathrm{CO}_{1}$ gene (Shearer et al., 2002; Erpenbeck et al., 2006). The multiple genotypes present in I. mirabilis indicate there is some balance between asexual and sexual reproduction at least in some part of the species history that has been maintained on different seamounts. This phenomenon has also been found in sponges (Zilberberg et al., 2006a), corals (Foster et al., 2007; Morrison et al., 2011) and ascidians (Perez-Portela \& Turon, 2008). For I. mirabilis it just means that it had recombination events at some point even prior to asexual transmission between the seamounts, which has been maintained in the population by asexual reproduction. The lack of subdivision indicates that either they all arose together and evolved very slowly with no continued divergence or they are mixing and interbreeding, with substantial gene flow between the seamounts. 
Molecular studies of other sponges have detected strong spatial structure and restricted gene flow, reproductively isolating the populations and thus increasing the chance for speciation (e.g. Duran et al., 2004a; Blanquer et al., 2009). Cryptic speciation amongst what was thought to be cosmopolitan species correlates with potential speciation, presumably due to the low dispersal abilities of many sponges (Wörheide et al., 2002b; Duran \& Rützler, 2006; Blanquer \& Uriz, 2007).

The lack of any genetic differences along latitudinal or longitudinal gradients indicates that currents around the seamounts potentially have eddies, caused by the unidirectional East Australian Current heading south-easterly, along the Western edge of the Norfolk Ridge (Ridgway \& Dunn, 2003). The two pairs of seamounts that have physically close pairs of seamounts, i.e. Zorro North and Zorro South, and Eponge North and Eponge South are not genetically close. This result is in contrast to Duran \& Rützler (2006) who only found virtually all of the populations of Chondrilla cf. nucula Schmidt, 1862, to be significantly different.

This study demonstrates that sponge populations on the deep seamounts studied here appear to be well connected. Despite being topographically small and isolated, the individual seamounts do not show high levels of population structure based on the molecular loci studied here. Not only do seamounts share the same sponge species they also share the same genotypes indicating a mixture of sexual and asexual reproductive strategies with better dispersal properties than previously thought.

\section{ACKNDWLEDGEMENTS}

We would like to thank Bertrand Richer de Forges and Thomas Schlacher for collection of the specimens and all the crew and scientific staff on the 'RV Alis' for the NORFOLK 2 Cruise. We would like to thank Emma Sherlock from the Natural History Museum for providing a fragment of the holotype of N. zingiberadix for examination. We would also like to thank Kathryn Hall, Monika Bryce and Jessica Worthington-Wilmer for technical advice, laboratory support and assistance. ME and DE would especially like to thank Dan Jackson, Alina Craige, Maja Adamska, Gabriele Büttner and Simone Schätzle for help with molecular work and Bernie Degnan for making space in his laboratory for cloning. We also would like to thank Liam Town and Carol Wicking for help with sequencing alignment and Andrea Crowther for suggestions about nested clade analysis. We would also like to thank Andrzej Pisera for his help with identification of one of the specimens of $N$. fulvodesmus. ME would also like to thank Judy Powell and Monique Grol for help with the manuscript.

\section{FINANCIAL SUPPDRT}

This research received in part funding of the Alfred P. Sloan Foundation, Census of Marine Life's Barcode of Life Initiative (\#2007-12-1-C4: 'DNA Barcoding of Marine Biodiversity (MarBOL)'). DE and GW acknowledge funding from the Deutsche Forschungsgemeinschaft projects (DFG) ER611/3-1, WO896/15-1.

\section{REFERENCES}

Atlas of Living Australia (ALA) (2014). Available at: http://bie.ala.org.au/ (accessed 16 April 2014).

Ayre D.J. and Willis B.L. (1988) Population structure in the coral Pavona cactus: clonal genotypes show little phenotypic plasticity. Marine Biology 99, 495-505.

Battershill C.N. and Bergquist P.R. (1990) The influence of storms on asexual reproduction, recruitment, and survivorship of sponges. In Rützler K. (eds) New perspectives in sponge biology. Washington, DC: Smithsonian Institution Press, pp. 397-403.

Bautista-Guerrero E., Carballo J.L. and Maldonado M. (2010) Reproductive cycle of the coral-excavating sponge Thoosa mismalolli (Clionaidae) from Mexican Pacific coral reefs. Invertebrate Biology 129, 285-296.

Bentlage B. and Worheide G. (2007) Low generic structuring among Pericharax heteroraphis (Porifera; Calcarea) population from the Great Barrier Reef (Australia), revealed by analysis of nrDNA and nuclear intron sequences. Coral Reefs 26, 807-816.

Blanquer A. and Uriz M.J. (2007) Cryptic speciation in marine sponges evidenced by mitochondrial and nuclear genes. A phylogenetic approach. Molecular Phylogenetics and Evolution 45, 392-397.

Blanquer A. and Uriz M.J. (2011) "Living together apart": the hidden genetic diversity of sponge populations. Molecular Biology and Evolution 28, 2435-2438.

Blanquer A., Uriz M.J. and Caujape-Castells J. (2009) Small-scale spatial genetic structure in Scopalina lophyropoda, and encrusting sponge with philopatric larval dispersal and frequent fission and fusion events. Marine Ecology Progress Series 380, 95-102.

Blanquer A., Uriz M.J. and Pascal M. (2005) Polymorphic microsatellite loci isolated from the marine sponge Scopalina lophyropoda (Demospongiae: Halichondrida). Molecular Ecology Notes 5, 466-468.

Bohonak A.J. (1999) Dispersal, gene flow and population structure. Quarterly Review of Biology 74, 21-45.

Boisselier-Dubayle M.C. (1999) Genetic relationships between marine and marginal-marine populations of Cerithium species from the Mediterranean Sea. Marine Biology 135, 671-682.

Bond C. and Harris A.K. (1988) Locomotion of sponges and its physical mechanism. Journal of Experimental Zoology 246, 271-284.

Borchiellini C., Alivon E. and Vacelet J. (2004) The systematic position of Alectona (Porifera, Demospongiae): a tetractinellid sponge. Bollettino dei Musei e degli Istituti Biologici della Università di Genova 68, 209-217.

Buss L.W. (1982) Somatic cell parasitism and the evolution of somatic tissue compatibility. Proceedings of the National Academy of Sciences USA 79, 5337-5341.

Calderon I., Ortega N., Duran S., Becerro M.A., Pascal M. and Turon X. (2007) Finding the relevant scale: clonality and genetic structure in a marine invertebrate (Carme crambe, Porifera). Molecular Ecology 16, 1799-1810.

Clark M.R., Schlacher T.A., Rowden A.A., Stocks K.I. and Consalvey M. (2012) Science priorities for seamounts: research links to conservation and management. PLoS ONE, e29232. doi:10.1371/journal.pone. 0029232.

Collin R. (2001) The effects of mode of development on phylogeography and population structure of North Atlantic Crepidula (Gastropoda: Calyptraeidae). Molecular Ecology 10, 2249-2262.

Dailianis T., Tsigenopoulos C.S., Dounas C. and Voultsiadou E. (2011) Genetic diversity of the imperilled bath sponge Spongia officinalis Linnaeus, 1759 across the Mediterranean Sea: patterns of population 
differentiation and implications for taxonomy and conservation. Molecular Ecology 20, 3757-3772.

Duran S., Giribet G. and Turon X. (2004a) Phylogeographical history of the sponge Crambe crambe (Porifera, Poecilosclerida): range expansion and recent invasion of the Macaronesian islands from the Mediterranean Sea. Molecular Ecology 13, 109-122.

Duran S., Pascal M., Estoup A. and Turon X. (2004c) Strong population structure in the marine sponge Crambe crambe (poecilosclerida) as revealed by microsatellite markers. Molecular Ecology 13, 511-522.

Duran S., Pascal M., Estoup A. and Turon X. (2002) Polymorphic microsatellite loci in the sponge Crambe crambe (Porifera: Poecilosclerida) and their variation in two distant populations. Molecular Ecology Notes 2, 478-480.

Duran S., Pascual M. and Turon X. (2004b) Low levels of genetic variation in mtDNA sequences over the western Mediterranean and Atlantic range of the sponge Crambe crambe (Poecilosclerida) Crambe crambe (Poecilosclerida). Marine Biology 144, 31-35.

Duran S. and Rützler K. (2006) Ecological speciation in a Caribbean marine sponge. Molecular Phylogenetics and Evolution 40, 292-297.

Edgar R.C. (2004) MUSCLE: multiple sequence alignment with high accuracy and high throughput. Nucleic Acids Research 32, $1792-1797$.

Ereskovsky A.V. (2010) The comparative embryology of sponges. Saint-Petersburg: Springer.

Ereskovsky A.V. and Tokina D.B. (2007) Asexual reproduction in homoscleromorph sponges (Porifera; Homoscleromorpha). Marine Biology $151,425-434$

Erpenbeck D., Hooper J.N.A. and Wörheide G. (2006) CO1 phylogenies in diploblasts and the 'Barcoding of Life' - are we sequencing a suboptimal partition? Molecular Ecology Notes 6, 550-553.

Excoffier L., Laval G. and Schneider S. (2005) Arlequin ver. 3.0: an integrated software package for population genetics data analysis Evolutionary Bioinformatics Online 1, 47-50.

Foster N.L., Iliana B.B. and Mumby P.J. (2007) Sexual vs. asexual recombination in an ecosystem engineer: the massive coral Montastrea annularis. Journal of Animal Ecology 76, 384-391.

Fromont J. and Bergquist P.R. (1994) Reproductive biology of three sponge species of the genus Xestospongia (Porifera: Demospongiae: Petrosiida) from the Great Barrier Reef. Coral Reefs 13, 119-126.

Galtier N., Gouy M. and Gautier C. (1996) SEAVIEW and PHYLO_WIN: two graphic tools for sequence alignment and molecular phylogeny. Computer Applications in the Biosciences 12, 543-548.

Hall K.A., Ekins M.G. and Hooper J.N.A. (2014) Two new desma-less species of Theonella Gray, 1868, (Demospongiae: Astrophorida: Theonellidae) from the Great Barrier Reef, Australia, and a re-evaluation of one species assigned previously to Dercitus Gray, 1867. Zootaxa 3814, 451-477.

Hall T.A. (1999) Bioedit: a user friendly biological sequence alignment editor and analyis program for Windows 95/98/NT. Nuclei Acids Symposium Series 41, 95-98.

Hooper J.N.A. and Van Soest R.W.M. (2002) Systema Porifera. A guide to the classification of sponges. New York: Kluwer Academic/Plenum Publishers.

Hoshino S., Saito D.S. and Fujita T. (2008) Contrasting genetic structure of two Pacific Hymeniacidon species. Hydrobiologia 603, 313-326.

Hughes T.P., Ayre D.J. and Connell J.H. (1992) Evolutionary ecology of corals. Trends in Ecology and Evolution 40, 357-365.

Jablonski D. (1986) Larval ecology and macroevolution in marine invertebrates. Bulletin of Marine Science 39, 565-587.
Jackson J.B.C. (1986) Modes of dispersal of clonal benthic invertebrates: consequences for species' distributions and genetic structure of local populations. Bulletin of Marine Science 39, 588-606.

Kaye H.R. (1990) Reproduction in West Indian commercial sponges: oogenesis, larval development, and behaviour. In Rützler K. (eds) New perspectives in sponge biology. Washington, DC: Smithsonian Institution Press, pp. 161-435.

Kelly M. (2007) The marine fauna of New Zealand: Porifera: lithistis Demospongiae (Rock Sponges). NIWA Biodiversity Memoir 121, $1-100$.

Klautau M., Russo C.A.M., Lazoski C., Boury-Esnault N., Thorpe J.P. and Solé-Cava A.M. (1999) Does cosmopolitanism result from overconservative systematics? A case study using the marine sponge Chondrilla nucula. Evolution 53, 1414-1422.

Kyle C.J. and Boulding E.G. (2000) Comparative population genetic structure of marine gastropods (Littorina spp.) with and without pelagic larval dispersal. Marine Biology 137, 835-845.

Le Goff-Vitry M.C., Pybus O.G. and Rogers A.D. (2004) Genetic structure of the deep-sea coral Lophelia pertusa in the northeast Atlantic revealed by microsatellites and internal transcribed spacer sequences. Molecular Ecology 13, 537-549.

Leong W. and Pawlik J.R. (2010) Fragments of propagules? Reproductive tradeoffs among Callyspongia spp. from Florida coral reefs. Oikos 119, $1417-1422$.

Lévi C. (1991) Lithistid sponges from the Norfolk Rise. Recent and Mesozoic genera. In Reitner J. and Keupp H. (eds) Fossil and recent sponges. Berlin: Springer-Verlag, pp. $72-82$.

Lévi C. and Lévi P. (1983) Éponges Tétractinellides et Lithistides bathyales de Nouvelle-Calédonie. Bulletin du Muséum National d'Histoire Naturelle, Paris (4) 5, 101-168.

Longo C., Pontassuglia C., Corriero G. and Gaino E. (2012) Life-cycle traits of Paraleucilla magna, a calcerous sponge invasive in a coastal Mediterranean basin. PLoS ONE 7, 1-12.

Lopez J.V., Peterson C.L., Willoughby R., Wright A.E., Enright E., Zoladz S., Reed J.K. and Pomponi S.A. (2002) Characterization of genetic markers for in vitro cell line identification of the marine sponge Axinella corrugata. Journal of Heredity 93, 27-36.

Maldonado M. (1998) Do chimeric sponges have improved chances of survival? Marine Ecology Progress Series 164, 301-306.

Maldonado M. (2004) Choanoflagellates, choanocytes, and animal multicellularity. Invertebrate Biology 123, 231-242.

Maldonado M. and Bergquist P.R. (2002) Phylum porifera. In Young C. (eds) Atlas of marine invertebrate larvae. San Diego, CA: Academic Press, pp. 21-49.

Maldonado M. and Riesgo A. (2008) Reproduction in the Phylum Porifera: a synoptic overview. Treballs de la SCB 59, 29-49.

Maldonado M. and Uriz M.J. (1999a) Sexual propagation by sponge fragments. Nature 398, 476 .

Maldonado M. and Uriz M.J. (1999b) An experimental approach to the ecological significance of microhabitat-scale movement in an encrusting sponge. Marine Ecology Progress Series 185, 239-255.

Meyer C.P., Geller J.B. and Paulay G. (2005) Fine scale endemism on coral reefs: archipelagic differentiation in turbinid gastropods. Evolution 59, 113-125.

Miller K.J. and Ayre D.J. (2004) The role of sexual and asexual reproduction in structuring high latitude populations of the reef coral Pocillopora damicornis. Heredity 92, 557-568.

Morrison C.L., Ross S.W., Nizinski M.S., Brooke S., Jarnegren J., Waller R.G., Johnson R.L. and King T.L. (2011) Genetic 
discontinuity among regional populations of Lophelia pertusa in the North Atlantic Ocean. Conservation Genetics 12, 713-729.

Neigel J.E. and Avise J.C. (1983) Histocompatibility bioassays of population structure in marine sponges. Clonal structure in Verongia longissima and Iotrochota birotulata. Journal of Heredity 74, 134-140.

Nichols S.A. and Barnes P.A.G. (2005) A molecular phylogeny and historical biogeography of the marine sponge genus Placospongia (Phylum Porifera) indicate low dispersal capabilities and widespread crypsis. Journal of Experimental Marine Biology and Ecology 323, $1-15$.

Perez-Portela R. and Turon X. (2008) Cryptic divergence and strong population structure in the colonial invertebrate Pycnoclavella communis (Ascidiacea) inferred from molecular data. Zoology 111, 163-178

Pisera A. and Lévi C. (2002) 'Lithistid' Demospongiae. In Hooper J.N.A. and Van Soest R.W.M. (eds) Systema Porifera: a guide to the classification of sponges. New York: Kluwer Academic/Plenum Publishers.

Puill-Stephan E., Willis B.L., van Herwerden L. and van Oppen J.H. (2009) Chimerism in wild adult populations of the broadcast spawning coral Acropora millepora on the Great Barrier Reef. PLOS ONE 4, e7751.

Reiswig H.M. (1973) Population dynamics of three Jamaican Demospongiae. Bulletin of Marine Science 23, 191-226.

Richer de Forges B., Koslow J.A. and Poore G.C.B. (2000) Diversity and endemism of the benthic seamount fauna in the southwest Pacific Nature 405, 944-947.

Ridgway K.R. and Dunn J.R. (2003) Mesoscale structure of the mean East Australian Current System and its relationship with topography. Progress in Oceanography 56, 189-222.

Samadi S., Bottan L., Macpherson E. and Richer de Forges B. and Boisselier M.C. (2006) Seamount endemism questioned by the geographic distribution and population genetic structure of marine invertebrates. Marine Biology 149, 1463-1475.

Samadi S., Schlacher T. and Richer de Forges B. (2007) Seamoun benthos. In Pitcher T.J., Horato T., Hart P.J.B., Clark M.R., Hagger N. and Santos R.S. (eds) Seamounts: ecology, fisheries and conservation. Carlton: Blackwell Publishing.

Sambrook J., Fritsch E.F. and Maniatis T. (1989) Molecular cloning: $a$ laboratory manual, 2nd edn. Cold Spring Harbor, NY: Cold Spring Harbor Laboratory Press.

Sarà A., Cerrano C. and Sara M. (2002) Viviparious development in the Antarctic sponge Stylocordyla borealis Loven, 1868. Polar Biology 25, 425-431.

Schlacher-Hoenlinger M.A., Pisera A. and Hooper J.N.A. (2005) Deep-sea "lithistid" assemblages from the Norfolk Ridge (New Caledonia), with description of seven new species and a new genus (Porifera, Demospongiae). Zoosystema 27, 649-698.

Schmitt S., Hentschel U., Zea S., Dandekar T. and Wolf M. (2005) ITS-2 and $18 \mathrm{~S}$ gene phylogeny of Aplysiniidae (Verongida, Demospongiae). Journal of Molecular Evolution 60, 237-336.

Schuster A., Erpenbeck D., Pisera A., Hooper J.N.A., Bryce M., Fromont J. and Wörheide G. (2015) Deceptive desmas: molecular phylogenetics suggests a new classification and uncovers convergent evolution of lithistid demosponges. PLoS ONE 10, e116038.

Shearer T.L., Van Oppen M.J.H., Romano S.L. and Wörheide G. (2002) Slow mitochondrial DNA sequence evolution in the Anthozoa (Cnidaria). Molecular Ecology 11, 2475-2487.

Silva E.P. and Russo C.A.M. (2000) Techniques and statistical data analysis in molecular population genetics. Hydrobiologia 420, 119-135.
Sommerfeldt A.D., Bishop J.D.D. and Wood C.A. (2003) Chimerism following fusion in a clonal ascidian (Urochordata). Biological Journal of the Linnean Society 79, 182-192.

Stamatakis A. (2006) RAxML-VI-HPC: maximum likelihood-based phylogenetic analyses with thousands of taxa and mixed models. Bioinformatics 22, 2688-2690.

Teixido N., Gili J.P., Uriz M.J., Gutt J. and Arntz W.E. (2006) Observations of asexual reproductive strategies in Antarctic hexactinellid sponges from ROV video records. Deep Sea Research II 53, 972-984.

Todd C.D., Lambert W.J. and Thorpe J.P. (1998) The genetic structure of intertidal populations of two species of nudibranch mollusks with planktotrophic and pelagic lecithotrophic larval stages: are pelagic larvae 'for' dispersal? Journal of Experimental Marine Biology and Ecology 228, 1-28.

Uriz M.J. and Turon X. (2012) Sponge ecology in the molecular era. Advances in Marine Biology 61, 345-410.

Vargas S., Erpenbeck D., Schuster A., Sacher K., Büttner G., Schätzle S. and Wörheide G. (2010) A high-throughput, low-cost Porifera DNA barcoding pipeline. In VIII. World Sponge Conference. Girona, Spain.

Whalan S., De Nys C., Smith-Keune R., Evans B.R., Battershill C.N. and Jerry D.R. (2008) Low genetic variability within and among populations of the brooding sponge Rhopaloeides odorabile on the central Great Barrier Reef. Aquatic Biology 3, 111-119.

Wörheide G. (2006) Low variation in partial cytochrome oxidase subunit i $(\mathrm{CO} 1)$ across mitochondrial sequences in the coralline demosponge Astrosclera willeyana across the Indo-Pacific. Marine Biology 148, 907-912.

Wörheide G., Degnan B.M., Hooper J.N.A. and Reitner J. (2002b) Biogeography and taxonomy of the Indo-Pacific reef cave dwelling coralline demosponge Astrosclera 'willeyana': new data from nuclear internal transcribed spacer sequences. In Moosa K.M., et al. (eds) Proceedings of the 9th International Coral Reef Symposium, Bali, Indonesia 23-27 October 200o. State Ministry for the Environment, Indonesia, Indonesian Institute of Sciences \& The International Society for Reef Studies, pp. 339-345.

Wörheide G., Epp L.S. and Macis L. (2008) Deep genetic divergences among Indo-Pacific populations of the coral reef sponge Leucetta chagosensis (Leucettidae): founder effects, vicariance, or both? BMC Evolutionary Biology 8, 1-18.

Wörheide G. and Erpenbeck D. (2007) DNA taxonomy of sponges progress and perspectives. Journal of the Marine Biological Association of the United Kingdom 87, 1629-1633.

Wörheide G., Hooper J. and Degnan B.M. (2002a) Phylogeography of western Pacific Leucetta 'chagosensis' (Porifera: Calcarea) from ribosomal DNA sequences: implications for population history and conservation of the Great Barrier Reef World Heritage Area (Australia). Molecular Ecology 11, 1753-1768.

Wörheide G., Nichols S.A. and Goldberg J. (2004) Intragenomic variation of the rDNA internal transcribed spacers in sponges (Phylum Porifera): implications for phylogenetic studies. Molecular Phylogenetics and Evolution 33, 816-830.

Wörheide G., Solé-Cava A.M. and Hooper J.N.A. (2005) Biodiversity, molecular ecology and phylogeography of marine sponges: patterns, implications and outlooks. Integrative and Comparative Biology 45 , $377-385$.

Wulff J.L. (1990) Patterns and processes of size change in Caribbean demosponges of branching morphology. In Rützler K. (eds) New perspectives in sponge biology. Washington, DC: Smithsonian Institution Press, pp. 425-435. 
Wulff J.L. (1991) Asexual fragmentation, genotype success and population dynamics of erect branching sponges. Journal of Experimental Marine Biology and Ecology 149, 227-247.

Xavier J.R., Rachello-Dolmen P.G., Parra-Velandia F., Schönbeg C.H.L., Brewer J.A.J. and Van Soest R.W.M. (2010) Molecular evidence of cryptic speciation in the "cosmopolitan" excavating sponge Cliona celata (Porifera, Clionaidae). Molecular Phylogenetics and Evolution 56, 13-20.

Zilberberg C., Maldonado M. and Solé-Cava A.M. (2006b) Assessment of the relative contribution of asexual propagation in a population of the coral-excavating sponge Cliona delitrix from the Bahamas. Coral Reefs 25, 297-301.

and
Zilberberg C., Solé-Cava A.M. and Klautau M. (2006a) The extent of asexual reproduction in sponges of the genus Chondrilla (Demospongiae: Chondrosida) from the Caribbean and the Brazilian coasts. Journal of Experimental Marine Biology and Ecology 336, 211-220.

\section{Correspondence should be addressed to:}

M. Ekins

Queensland Museum, PO Box 3300, South Brisbane 4101, Brisbane, Queensland, Australia email: m.ekins@qm.qld.gov.au 